\title{
Review of in vivo optical molecular imaging and sensing from $x$-ray excitation
}

\author{
Brian W. Pogue $\odot$, , ,b, $*$ Rongxiao Zhang, ${ }^{\text {a,b }}$ Xu Cao $\odot,{ }^{\mathrm{a}}$ \\ Jeremy Mengyu Jia $\odot,{ }^{c}$ Arthur Petusseau, ${ }^{a}$ Petr Bruza $\odot,{ }^{a}$ and \\ Sergei A. Vinogradov ${ }^{\mathrm{d}, \mathrm{e}}$ \\ ${ }^{a}$ Thayer School of Engineering at Dartmouth, Hanover, New Hampshire, United States \\ ${ }^{\mathrm{b}}$ Geisel School of Medicine at Dartmouth, Lebanon, New Hampshire, United States \\ ${ }^{c}$ Stanford University School of Medicine, Department of Radiation Oncology, Palo Alto, \\ California, United States \\ ${ }^{\mathrm{d}}$ University of Pennsylvania, Perelman School of Medicine, Department of Biochemistry and \\ Biophysics, Philadelphia, Pennsylvania, United States \\ ${ }^{\mathrm{e}}$ University of Pennsylvania, School of Arts of Sciences, Department of Chemistry, Philadelphia, \\ Pennsylvania, United States
}

\begin{abstract}
Significance: Deep-tissue penetration by $\mathrm{x}$-rays to induce optical responses of specific molecular reporters is a new way to sense and image features of tissue function in vivo. Advances in this field are emerging, as biocompatible probes are invented along with innovations in how to optimally utilize x-ray sources.

Aim: A comprehensive review is provided of the many tools and techniques developed for x-rayinduced optical molecular sensing, covering topics ranging from foundations of $\mathrm{x}$-ray fluorescence imaging and $\mathrm{x}$-ray tomography to the adaptation of these methods for sensing and imaging in vivo.
\end{abstract}

Approach: The ways in which x-rays can interact with molecules and lead to their optical luminescence are reviewed, including temporal methods based on gated acquisition and multipoint scanning for improved lateral or axial resolution.

Results: While some known probes can generate light upon x-ray scintillation, there has been an emergent recognition that excitation of molecular probes by $\mathrm{x}$-ray-induced Cherenkov light is also possible. Emission of Cherenkov radiation requires a threshold energy of $\mathrm{x}$-rays in the high $\mathrm{kV}$ or MV range, but has the advantage of being able to excite a broad range of optical molecular probes. In comparison, most scintillating agents are more readily activated by lower keV x-ray energies but are composed of crystalline inorganic constituents, although some organic biocompatible agents have been designed as well. Methods to create high-resolution structured x-rayoptical images are now available, based upon unique scanning approaches and/or a priori knowledge of the scanned x-ray beam geometry. Further improvements in spatial resolution can be achieved by careful system design and algorithm optimization. Current applications of these hybrid x-ray-optical approaches include imaging of tissue oxygenation and $\mathrm{pH}$ as well as of certain fluorescent proteins.

Conclusions: Discovery of x-ray-excited reporters combined with optimized x-ray scan sequences can improve imaging resolution and sensitivity.

(C) The Authors. Published by SPIE under a Creative Commons Attribution 4.0 Unported License. Distribution or reproduction of this work in whole or in part requires full attribution of the original publication, including its DOI. [DOI: 10.1117/1.JBO.26.1.010902]

Keywords: Cerenkov; emission; fluorescence; phosphorescence; radioluminescence; scintillation.

Paper 200308VR received Sep. 21, 2020; accepted for publication Nov. 24, 2020; published online Jan. 1, 2021.

*Address all correspondence to Brian W. Pogue, Brian.W.Pogue@dartmouth.edu 


\section{Introduction}

Optical molecular imaging and sensing from x-ray excitation utilizes a fundamentally different type of interaction and sensing approach to excite optical reporters in biological tissues and detect and localize the emission. The biological utility and goals of x-ray-based sensing to better understand tissue physiology and pathophysiology are the driving motivations that underpin technical advances in this area. The major benefits of x-rays as a probe excitation source are (i) the high penetrance and (ii) the wide availability and acceptance of x-ray sources in biomedical imaging. When compared to other methods of molecular sensing in tissue, the strengths of $\mathrm{x}$-ray-based molecular sensing may be less clear, because this methodology only began emerging during the last decade. ${ }^{1-3}$ However, the ability to sense through tissue using traditional x-ray sources, while still using optical molecular contrast, presents potential advantages in depth penetrance and spatial resolution. Optical sensing provides superior molecular sensitivity to x-raybased contrast methods, because $\mathrm{x}$-ray contrast is generally based upon the photoelectric effect, with peak attenuation in the keV energy range, ${ }^{4}$ and $\mathrm{x}$-ray contrast agents usually need to be present in high levels, near millimolar quantities in tissue making them only useful for imaging blood volume and leakage. The field of x-ray-based molecular sensing benefits from an extraordinarily large range of detectors and sensors for optical emission that have sensitivity to the single photon level, making the detection side of the sampling potentially very efficient.

Perhaps the most scientifically enticing part of this methodology is the concept of utilizing one radiation source (i.e., $\mathrm{x}$-rays) as the excitation probe, combined with another radiation type as a signal. This conceptual framework for developing a hybrid imaging modality is illustrated in Fig. 1 for several possible emission radiation types. X-ray activations with emission via pathways that are detectable through tissue are illustrated, including (a) x-ray-induced fluorescence, (b) x-ray-induced optical luminescence (the focus of this review), (c) x-ray-induced electromagnetic induction, and (d) x-ray-induced acoustics. While not all these approaches are reviewed extensively here, the ideal characteristics of such a hybrid method include:

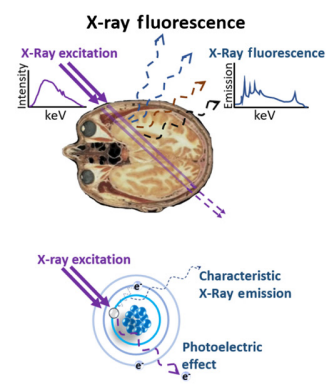

(a)
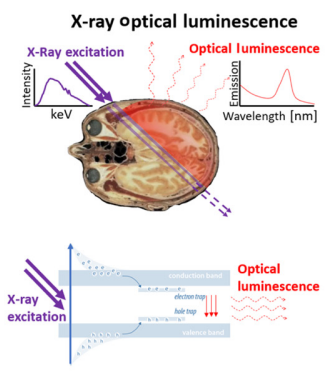

(b)
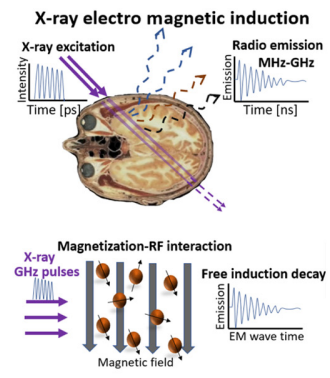

(c)

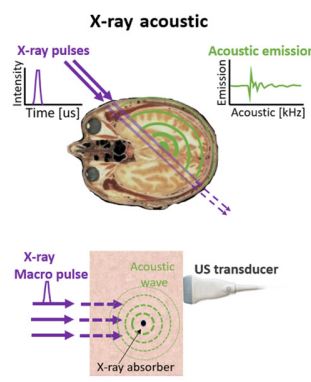

(d)

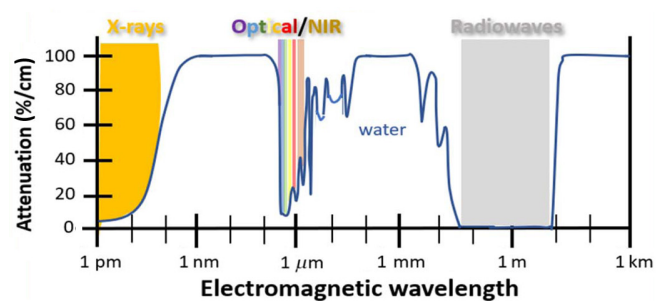

(e)

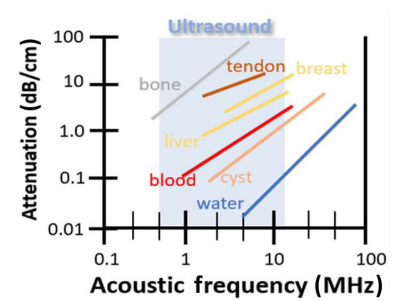

(f)

Fig. 1 Schematic of the different detection schemes for probes, including (a) XRF where x-rays are both the excitation and emission, (b) x-ray optical luminescence where the output emission signal is optical from either scintillation or Cherenkov processes, (c) $\mathbf{x}$-ray electromagnetic induction where there is either nuclear moment or electromagnetic change induced in the tissue, such that the output is a radiofrequency signal, and (d) x-ray acoustic where the output is induced by localized heating causing an ultrasound transient. As follows in (e) the attenuation spectrum of water is shown for electromagnetic radiation and in $(f)$ the attenuation spectrum of acoustic frequencies is shown in tissue. 
i. unique molecular probe, able to bind with high affinity to biological targets;

ii. high contrast or specificity via high signal-to-background ratio;

iii. an emission signal type that can be detected with high signal-to-noise ratio; and

iv. an excitation radiation with high penetrance for imaging through tissue $(1 / \mu \approx d$, where $\mu$ is the exponential attenuation coefficient and $d$ is tissue thickness).

The emission signal types are illustrated in Fig. 1, with the attenuation values shown in Figs. 1(e) and $1(\mathrm{f})$.

In the case of optical emission, the single largest attraction is that optical molecular probes comprise arguably the most developed and diverse group of sensors for biological imaging. There are thousands of optical probes, and a number of them are commercially available for use in preclinical imaging. Consequently, preclinical optical imaging systems are the most widely used for whole body animal imaging, ${ }^{5}$ and imaging of tissue function and pathology with many types of optical stains is widely used both in vivo and ex vivo. The second major attraction for optical emission detection is its very high sensitivity, down to the single photon level. However, because of tissue scattering and absorption, there is exponential attenuation of optical signals with depth into tissue, although imaging through several centimeters of tissue is possible. The combination of a scanning x-ray excitation with detection of optical emission can circumvent the resolution limitations of diffuse optical imaging, while having the advantage of optical sensitivity and broad selection of optical imaging agents. Alternatively, combinations of optical sensing with other structural imaging tools, such as ultrasound, MRI, or computed tomography (CT), are also a commercially available paradigm.

The choices of x-ray sources, type, and mode of detectors are also quite diverse. The energy range of the x-rays needed depends upon the type of probe and its physical interaction mechanisms, as outlined in Fig. 2. Interaction mechanisms range from low keV energy x-rays, where the interaction with the probe is via the photoelectric effect, up to low $\mathrm{MeV}$ energies, where generation of Cherenkov radiation is the dominant mode to excite the probe. Overall, the following choices need to be considered: (i) x-ray source, (ii) molecular probe, (iii) emission detector, and (iv) the methodology of image recovery. These topics are reviewed as follows, with the focus on the advantages and disadvantages of existing approaches and on how the aforementioned choices are interconnected. As the technology progresses, it is imperative to think about the motivations, driving factors, and capabilities. This review begins with a historical summary from the origins of $\mathrm{x}$-ray fluorescence (XRF) methods towards $\mathrm{x}$-ray optical luminescence methods, with a focus on molecular sensitivity and utility, and then ends on a focus of what is needed for advancing the field capabilities.

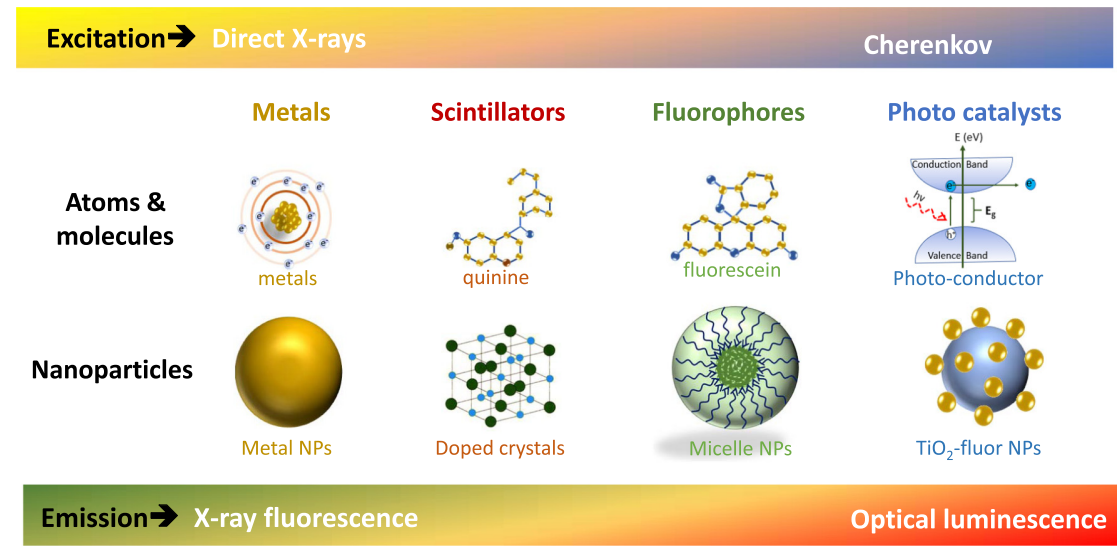

Fig. 2 Illustration of the four major categories of probes possible with $x$-ray excitation, roughly categorized in terms of their capabilities for (upper bar) direct x-ray excitation or by secondary Cherenkov emission and (lower bar) by their emission capabilities transitioning from XRF to $x$-ray luminescence. Each category has both molecular (top row) and nanoparticle (bottom row) forms which are $\mathrm{x}$-ray active. 


\section{X-Ray Fluorescence to X-Ray Optical Luminescence}

The basic principles of using X-rays to sample tissue comes from the successful origins of XRF sensing, where the concentration of trace metals such as $\mathrm{Fe}, \mathrm{Zn}, \mathrm{Cu}, \mathrm{Hg}$, and $\mathrm{Se}$ are quantified within tissue by detection of the secondary characteristic x-rays emitted when irradiating the sample with an excitation of incident X-rays or gamma rays. ${ }^{6-8}$ In this case, the word "fluorescence" is used to imply the emission of these specific x-rays, having narrow energy bands specific to the inner electron shell transitions of the metals. The actual interaction between the x-ray beam and the background medium produces a dominance of Compton scattered photons, which contribute mostly to background. XRF imaging has been developed as a CT as well, ${ }^{9-12}$ and more recently extensively developed as a microscopy tool for sliced tissue imaging of trace elements. ${ }^{13}$ One of the most striking things about XRF is that while there is high specificity to metals, the sensitivity is poor when compared to most in vivo molecular imaging tools. The limits of detection are near $0.1 \mathrm{mg} / \mathrm{g}$, placing this near the high millimolar range of sensitivity, while for comparison both fluorescence and nuclear medicine methods have concentration sensitivity of nanomolar to picomolar. ${ }^{8}$ As with optical methods, the sensitivity in thin tissues can be substantially higher than with in vivo use. ${ }^{14,15}$ The issues that limit detection in XRF are the (i) high background from Compton scatter, (ii) high detector noise, and (iii) low detector capture efficiency of the signals. Tissue sensing and imaging nearly necessitate the use of monoenergetic gamma rays or synchrotron x-ray sources to suppress the non-specific background and achieve higher signal to noise. ${ }^{16,17}$ The strength of the synchrotron approach is that the background is low and most of the stronger emission lines are clearly separated from one another.

From these origins in XRF over a few decades, an exciting direction of development was to utilize other excitation modes, or other emitted signals, that might provide molecular-specific signals. Additionally, these developments might provide a wider range of available signals, ${ }^{18}$ in the transition from strictly $\mathrm{x}$-ray contrast agents to other radioluminescence excitation modes. This is illustrated in Fig. 2. For any probe agent, either molecule or nanoparticle, molecular specificity can be gained in one of two ways, either directly by probing the atom/molecule/nanoparticle itself that localizes somewhere or by using molecular target specificity, where an XRF signal that is visible is from the tag on the carrier molecule. While some metal atoms can be excellent tags, higher signal levels might be achieved by use of nanoparticles that have higher cross section per particle. But more generally, this transition brings with it the need to examine which types of molecules or molecular complexes would provide sufficient or superior signal from x-ray excitation, and also which types of emission might provide maximal emissivity from tissue for high $\mathrm{S} / \mathrm{N}$ detection.

A natural transition then is to utilize a more sensitive detection mode that can reach down to the single photon level, such as either isotopic gamma emission in nuclear medicine or optical luminescence methods such as fluorescence or phosphorescence, to increase the signal level and/ or suppress the background and noise levels. ${ }^{18}$ To preserve tissue penetration, early attempts examined x-ray luminescence imaging from a range of red to infrared emitting phosphors, as this window of wavelengths has high transmission through tissue, albeit with significant diffuse scatter. Other emission windows could be the electromagnetic regions of $\mathrm{GHz}$ to $\mathrm{MHz}$, which have exceptionally low attenuation, although this remains to be explored fully. Alternatively, acoustic emission could be used, as is widely implemented in photoacoustics. This field is called $x$-ray acoustic imaging or radioacoustics. ${ }^{19-23}$ These modes of sensing the $\mathrm{x}$-ray interaction are illustrated in Fig. 2, with the incident and detection scheme on top and the physical mode of contrast illustrated on bottom. It is interesting, albeit hard, to compare the sensitivities of detection for each of these four possible modes of detection, because the range of interactions and the range of detectors are so diverse. The interaction sensitivity for $\mathrm{x}$-ray acoustic is generally low because the total amount of energy released by the radiation dose in tissue is low. However, ultrasound transducers are exquisitely well developed with high sensitivity, and so some preliminary studies have shown that this mode of detection is possible. Still the sensitivity of fluorescence emission detection can be orders of magnitude higher than acoustic detection in the setting of biological imaging, because of the ability of the detectors to capture single quanta of emission in optics. But the geometry and design of any detection system can alter this sensitivity by orders of magnitude. 


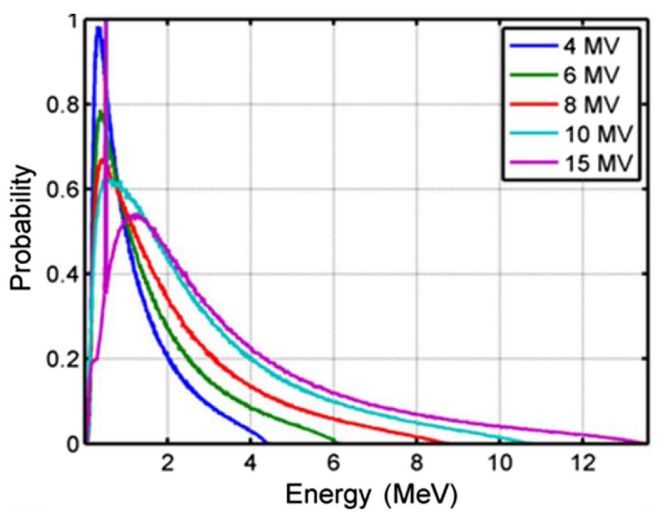

(a)

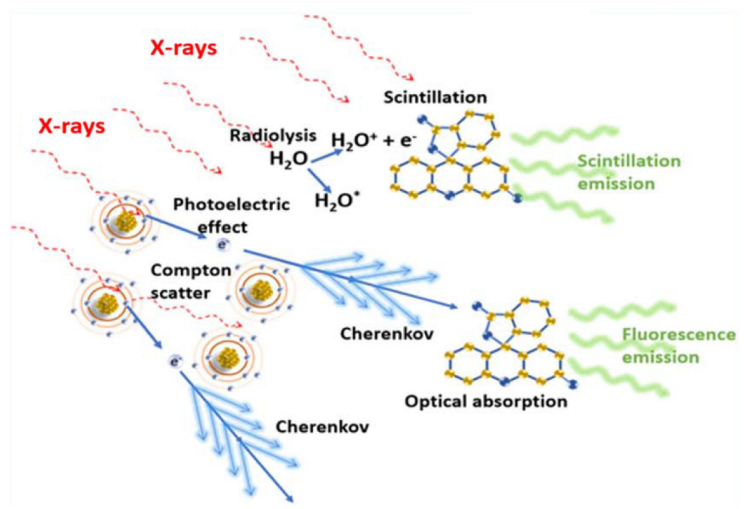

(c)

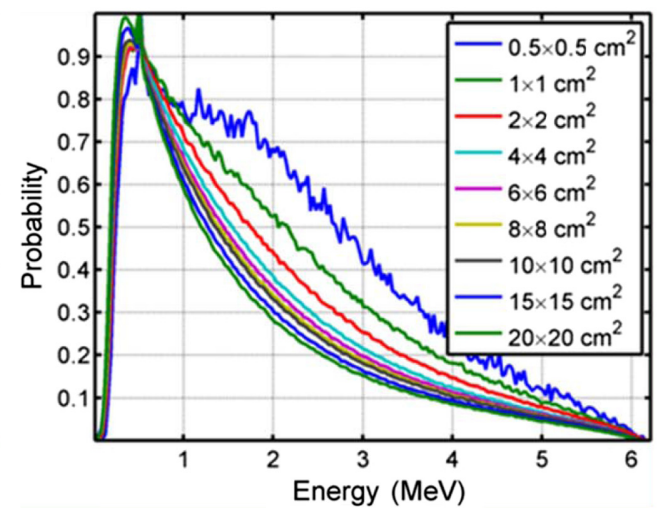

(b)

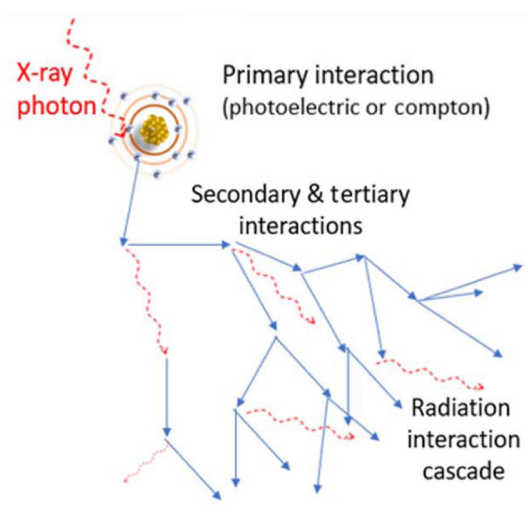

(d)

Fig. 3 X-ray energy spectra from Bremsstrahlung production via a Linac (a) for different energies, and the spectrum for $6 \mathrm{MV}$ at different beam diameters (b) showing higher energies at smaller beams. A schematic of the processes from $\mathrm{x}$-rays to optical emission is shown in (c) with either direct scintillation of a molecule mediated by a variety of radiolytic events in the medium or indirect transfer by a sequence of photoelectric or Compton emitted electrons generating Cherenkov light, which then yields optical photons absorbed by the molecule. The radiation-induced cascade is illustrated in (d) from a series of interaction events between secondary electrons and photons, leading to broad mechanisms of energy dissipation in the medium.

The excitation mode of an optically active contrast agent depends upon the mechanism of interaction with the radiation, as illustrated in Fig. 2, and the energy spectrum of the radiation source. While a synchrotron or isotope sources can produce monoenergetic beams of x-rays or gamma rays, most high yield practical sources of $\mathrm{x}$-rays are via the Bremsstrahlung effect from an electron beam impinging upon a target, producing a broad spectrum source, as illustrated in Figs. 3(a) and 3(b). Only a small fraction of the energy is given off as x-rays, and the spectrum is heavily weighted to the lowest energy photons. Thus, there is little specificity in the beam excitation from traditional x-ray sources, with a broad energy spectrum. The major modes of excitation, as illustrated in Fig. 3(c), are either:

1. Direct or indirect electronic excitation of a scintillator through the upper singlet states of pi electrons, resulting in eventual radiative decay. The complexity of processes depends upon the nature of both medium and scintillator, which determines their interactions. The end products of water hydrolysis (i.e., $\mathrm{H}^{*}, \mathrm{HO}^{*}, \mathrm{OH}^{-}, \mathrm{H}_{3} \mathrm{O}^{+}, \mathrm{H}_{2}$, and $\mathrm{H}_{2} \mathrm{O}_{2}$ ) dominate the interactions since water is in the largest concentration, and these can transfer energy with the scintillator. A well-known example of this is quinine, which appears to exhibit both scintillation and fluorescence; however, the interaction with the medium tends to be the dominating factor in this induction.

2. Electron soft collisions in the medium resulting in Cherenkov emission as part of the soft collision processes, which excites the molecules through direct singlet state absorption. 
This is proportional to the index of refraction of the medium for Cherenkov emission and then the overlap of the Cherenkov spectrum with the absorbance of the molecules. Almost all molecules with an absorption band in the visible with high emission yield would work for this method, although red absorbing molecules are more significant due to the blue absorption of Cherenkov by blood in tissue.

The overall efficiency of both these modes is generally low, although molecules with high quantum yield of emission, such as fluorescein, can make this process more favorable. The radiation cascade that occurs, Fig. 3(d), leads to a wide range of interaction mechanisms that can further decrease efficiency of any single pathway. Still the energy of a single x-ray photon $(1 \mathrm{MV})$ is $10^{6}$ higher than that of an optical photon $(1 \mathrm{eV})$, indicating that even a small efficiency process could lead to tens or hundreds of optical photons per x-ray photon.

\section{X-Ray-Induced Optical Tomography}

X-ray luminescence tomography was first postulated in 2010, showing that radioluminescent particles could be imaged from within tissue phantoms by scanning x-ray beams to excite them and capturing the emission of the signal., ${ }^{424}$ This was advanced to include modeling of the diffusive light transport, accounting for signal loss as a function of distance between the emission site and the light detection at the surface. The use of a partial angle approach to imaging is almost required for this type of work and several groups illustrated how this could be achieved in phantoms ${ }^{25-29}$ and in vivo. ${ }^{30,31}$ The major limitation in this aspect of work has been the sensitivity to mainly nanoscintillators used. The interaction mechanisms explored have largely been via direct excitation of the particle by the x-ray beam, where the reliance upon the photoelectric effect necessitates the use of high atomic number, $Z$, materials, and largely crystalline structures to gain sufficient radioluminescence yield from the process (see Fig. 2). The efficiency depends significantly upon the energy of x-rays, $E$, used because of the strong interaction cross section dependence of the photoelectric effect with energy, being $\sigma \approx Z^{4} / E^{3}$.

In comparison to direct $\mathrm{x}$-ray interaction, the process of Cherenkov radioluminescence provides a secondary mechanism for excitation. The strength of using Cherenkov is that it is produced throughout the volume, proportional to dose, and provides a broadband blue-white light source within the tissue. Although the weaknesses are that it is only produced from secondary electrons above the threshold of $220 \mathrm{keV}$ energy in tissue and with a yield about $1 \%$ of the total dose delivered. So, while there are attractive optical features of this excitation, it requires highenergy x-ray sources and yet produces a limited yield of light. Still, Cherenkov luminescence imaging has been demonstrated from isotopes in vivo as well as from linear accelerators used in radiation therapy. The latter provides a way to image tissue with higher fidelity because of the ability to scan the beam across the tissue and use image processing tools to recover highresolution images. The major benefit of this approach is that fluorescent and phosphorescent excitation can be achieved directly by absorption of the Cherenkov light by the molecular probe. These molecular probes are smaller, and many can be biocompatible, as are discussed as follows.

\section{X-Ray Sources, Beams, and System Design}

\subsection{X-Ray Sources and Beam Control}

One of the key values in x-ray-based molecular sensing is the concept that the position of the excitation source is known a priori and can be used in an image reconstruction process, such that even if the emission is blurred by optical scattering through tissue, there can be a high fidelity image recovered. This was initially demonstrated in x-ray optical luminescence tomography by Pratx et al., ${ }^{3}$ using keV x-ray imaging and image reconstruction. Diffusion modeling can be employed to reduce errors associated with the intensity of the luminescence emission being reduced by the tissue propagation. The geometries used directly follow those available for 
$\mathrm{x}$-ray CT because the source technology is derived from this. In the keV energy range, swept line scan beams or partial angle tomography is possible, ${ }^{12,29,32}$ and in MV photon beams from linear accelerators, multi-leaf collimators (MLC) and jaws are available to shape the beams dynamically. ${ }^{33}$ Full field imaging can work as well, albeit without significant axial resolution because of optical scatter in the tissue, but providing apparent high resolution in lateral imaging of the tissue surface..$^{34}$

\subsection{Temporal Acquisition}

The first studies of x-ray-induced optical luminescence based upon scintillation did not acquire in temporal sampling because the x-ray sources were continuous, and so there was no inherent value to temporal sampling. However, in Cherenkov-excited luminescence imaging, there is both inherent value in removal of the background Cherenkov excitation light, as well as the benefit of lock-in detection to the pulsed $\mathrm{x}$-ray source. Linear accelerators produced for clinical use most commonly have pulsed electron bunches that are accelerated in short 3- to 5-ms pulses, with a low repetition rate near 100 to $400 \mathrm{~Hz}$. This sampling is significantly slower than used for time-resolved fluorescence but fits with time-resolved phosphorescence methods, and so the first in vivo demonstrations have focused on imaging luminescence from oxygen sensors that have triplet states that are quenched on this timescale. Faster pulsed $\mathrm{x}$-ray sources exist though, such as small $\mathrm{x}$-ray sources for portable $\mathrm{x}$-rays ${ }^{20}$ or even large sources used for industrial pulsed radiography. ${ }^{35}$ The fastest commercially available pulsed x-ray sources are in the tens of nanoseconds range for pulse length, and the completeness of the fall off is usually uncharacterized or unspecified. Thus, fast time gating for things such as fluorescence from organic molecules, with lifetimes usually in the nanosecond range, would need a deconvolution for the x-ray-detector instrument response function, ${ }^{36,37}$ as is commonly done in time-correlated single photon counting work. $^{38}$

Temporal gating has been demonstrated with luminescence emission to suppress the Cherenkov light signal and allow for a nearly background-free sensing of the tissue. Single lymph node imaging was demonstrated by Zhang et al, ${ }^{33}$ using an oxygen sensing agent, and the lifetime recovery between 22 and 44 ms provided the ability to sense the local partial pressure of oxygen $\left(\mathrm{pO}_{2}\right)$. Sensing of other luminescent species is readily possible and Europium microspheres are commercially available agents for binding with targeting moieties that have a luminescence lifetime in the 100's of microseconds. ${ }^{39}$ Alternatively, silicone nanoparticles also have a long lived lifetime and can be used as a light signal generator with targeted delivery. ${ }^{40}$

\subsection{Lateral and Axial Spatial Resolution}

Perhaps one of the most undeveloped areas in x-ray-based molecular sensing is control of the $\mathrm{x}$-ray source for improvement in spatial resolution. Advances in conformal and adaptive radiotherapy have led to improved tools on a Linac to control beam dose deposition. The MLCs present on the output of the Linac have advanced to a very high degree of precision, where millimeter level accuracy in dose fall off can be achieved. This same precision can then be deployed where the lateral and axial extent of the beams are used to adjust the sampling of tissue. As mentioned above, lateral resolution is largely controlled by the MLCs and jaws of the Linac, but the axial resolution is determined by choices of the beam radiation type (electrons, photons, and protons) or beam depth of scanning, as illustrated in Fig. 4(a). Thus, the Linac MLCs provide a simple technological way to shape the beams for line scans, point scans, multi-point or multiline scans [see Fig. 4(a)], or even more generally a set of orthogonal basis functions in the source patterns for approaches such as compressed sensing. ${ }^{41,43,44}$

If the depth of penetration is to be restricted, it is feasible to use electron irradiation which has limited depth of penetration, and even apply a variable bolus or buildup region to the beam before the tissue, illustrated in Fig. 4(b), thereby allowing the depth of sampling to be varied externally to the Linac, just like an MLC adjusts the lateral component of the beam. The depth of sampling with electrons could be varied as they have the steepest fall off curve as compared to photons. Alternatively, in $\mathrm{keV}$ systems, various attempts have been shown to use x-ray focusing 


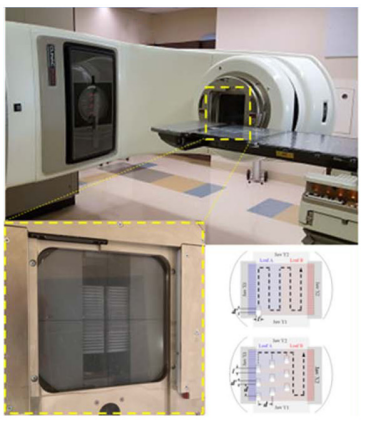

(a)

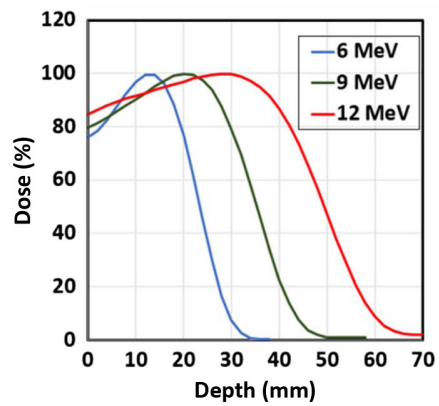

(b)

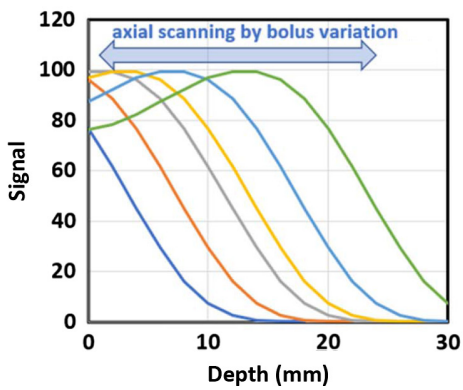

(c)

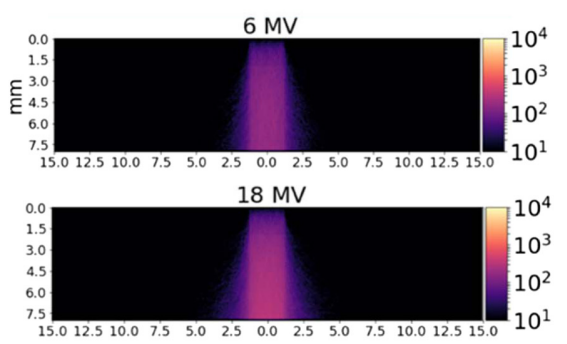

(d)

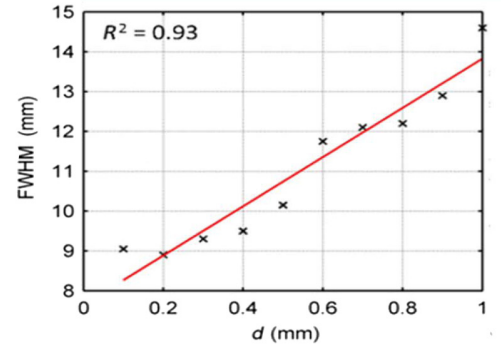

(e)

Fig. 4 The lateral spatial confines of the beam in tissue are determined by the Linac (a) beam shaping by the MLCs (photo inset below) with illustration of how the MLCs can be used for point raster scanning or multipoint scanning. ${ }^{41}$ (b) The depth of sensing is affected by the choice of radiation and beam energy, as illustrated by depth-dose curves for electron beams. The scan could be axially modulated in depth by variation of buildup bolus between the tissue and the beam, as illustrated in (c). Lateral resolution is limited by the ability to slice or focus the x-ray beam, as illustrated by Monte Carlo simulations of at 6- and 18-MV beams (d), where the beam is directed downward and the $X-Y$ axes show the $\mathrm{mm}$ dimensions into the tissue and the color bar is a Cherenkov intensity scale. ${ }^{42}$ The final lateral spatial resolution can be deconvolved with the beam width, show sensitivity to sub-millimeter objects, as illustrated in (e) where thin luminescent capillaries of varying diameter, $d$, were used to show sensitivity to resolve the full width at half maximum (FWHM) down to $0.2-\mathrm{mm}$ diameter. ${ }^{33}$ The FWHM is a convolution of the beam width with $d$.

mirrors or fiber optic tapers ${ }^{45-47}$ and as this technology evolves it might be feasible to limit lateral resolution with this focusing approach.

\subsection{Multiplexing of Signals}

Excitation with x-rays implicitly leads to a broadband signal that can excite more than one molecular probe. Both scintillation and Cherenkov-excited signals can occur, and it is possible to detect multiple emission signals, based either on temporal gating or wavelength separation of intensity. The excitation of multiple molecular dyes by Cherenkov has been shown, with optimal spectral windows being in the red, ${ }^{48,49}$ near-infrared, and short-wave infrared (SWIR) (also referred to as near-infrared window II, NIR-II) $)^{50-52}$ wavelength bands. In particular, SWIR emitting agents are often nanoparticulate and thereby optimally suited for direct x-ray excitation. ${ }^{50,53,54}$ There is a high volume of research in this area, and multicolor emitters are available. ${ }^{55,56}$ Detectors for SWIR wavelengths are often different than those for the red or NIR wavelengths, ${ }^{57}$ and so it is feasible to use separated detectors for parallelization or spectral decomposition through a spectrometer. ${ }^{58,59}$ Time-gated detection can be coupled with wavelength-based separation of the signals as well, to further maximize the ability to detect signals at the same time, and scintillation-based signals can be orders of magnitude faster than organic fluorescence, which in turn is orders of magnitude faster than phosphorescent signals. Careful sampling of the time-sequence of the x-ray pulses with the detector gating can optimize this. 


\section{Image Reconstruction}

\subsection{X-Ray Beam Location Used as Prior Spatial Information in Imaging}

X-ray photons experience much less scattering than optical radiation in tissue, and therefore $\mathrm{X}$-ray-induced radioluminescence largely originates within or very near the volume that is directly in the pathway of the scanning beam. The limits on resolution though can be defined by the $\mathrm{x}$-ray Compton scattering, which can happen both inside and outside the tissue. For keV sources, the scattering is usually not significant because it largely produces soft $\mathrm{x}$-rays that have very short penetration depth in tissue. This is true both outside the tissue and within the tissue. In the MeV approach, the Linac MLC (Fig. 4) provides a simple technological way to shape the beams for various scans as mentioned above, but also to induce x-ray scattering near the leaf boundaries, to some degree, depending on the specific leaf end structure. ${ }^{60,61}$ A similar situation exists within the tissue, where in Cherenkov-excited luminescence emission, most Cherenkov photons (in the UV-blue spectral range) have very short diffusion path $(<1 \mathrm{~mm})$ in the tissue due to the hemoglobin and water absorption. These properties constitute the key aspect of the x-rayinduced molecular imaging scheme, where the distribution of optical signal along the direction of scanning can be recovered by measuring total luminescence signal and considering that the signal all originated from the position of the scan beam in the tissue, used as prior information in a reconstruction algorithm.

\subsection{Diffusion Modeling of Cherenkov and Optical Photons}

The radiative transfer of photon diffusion through turbid media can be solved either numerically through Monte Carlo simulation or approximated analytically by the diffusion approximation. Monte Carlo solutions generally maintain high accuracy and wide applicability, ${ }^{62,63}$ and their use has been tremendously accelerated by computation on GPUs. ${ }^{64-69}$ There are extensions of this to model high-energy particle transport that can also be used, e.g., GEANT4, although with considerably more computational effort. ${ }^{70-73}$ The diffusion approximation provides a first-order approximation of transport over distances beyond a few millimeters, and it has been broadly applied in diffuse optical tomography. The limits to this approximation though are important, meaning that it must be applied only where the detected photons are scattered sufficiently to lose their original directions. This condition could be marginally met in Cherenkov imaging depending in the red and NIR wavelength bands. ${ }^{74,75}$ For example, the emission UV-blue spectral range that always predominates the measurement is significantly absorbed in most biological tissues, and so is rarely diffuse in transport. ${ }^{76,77}$

Cherenkov light is generally modeled in a comprehensive manner that involves distinct behavior of a broadband wavelength spectrum of photons peaked in the ultraviolet and decaying in intensity with an inverse square dependence upon the wavelength. ${ }^{78}$ Modeling of x-rayinduced Cherenkov as the excited light contains two coupled processes of excitation and emission, and explicitly would require modeling the x-ray interactions with Monte Carlo tools such as GEANT4. In practice, the Cherenkov/x-ray beam can be assumed to be non-diffuse source of light inside the tissue, to simply the process. The Monte Carlo codes that model radiationinduced light transport in biological media have been integrated into the GAMOS interface to GEANT4. ${ }^{73}$

\subsection{Typical Image Reconstructions: Advantages and Limitations}

Currently, image reconstructions could be coarsely classified into three categories: modelbased $^{79,80}$ direct deconvolution, ${ }^{43,81,82}$ and back-projection. ${ }^{32,83}$ The model-based approaches have the widest applicability to measurement geometry and excitation mechanisms. However, a model-based reconstruction can be inefficient and even ineffective when the target is close to or far from the boundary at the detection side. In the former case, a stringent forward model using either Monte Carlo or higher-accuracy approximations to exact radiation transport modeling is requisite; while in the latter case, any tiny perturbation and inconsistency of the optical properties could lead to erroneous reconstructions on both the intensity and location. 


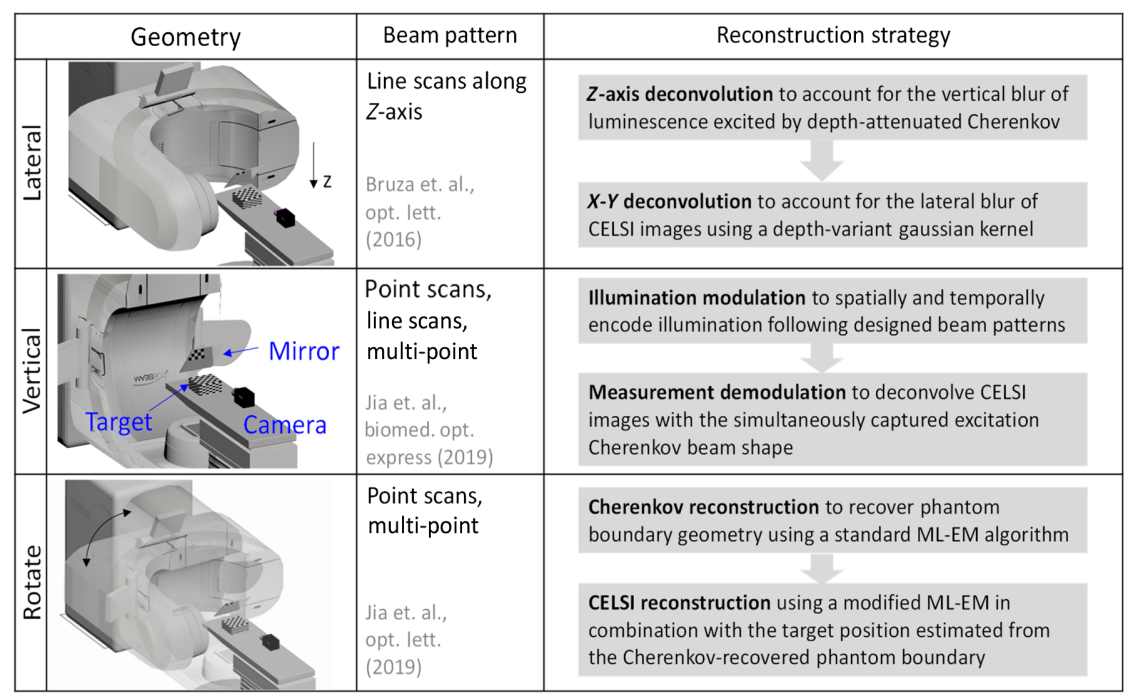

Fig. 5 Cherenkov radioluminescence measurement geometries based on Linac MLC delivery and corresponding non-model-based image reconstructions. Laterally scanned imaging (top) sends sheet-shaped $\mathrm{x}$-ray beams to measure local luminescence emission that is then deconvolved with the kernels derived from a diffusion approximation. Vertically scanned imaging (middle) spatially and temporally modulates the Cherenkov illumination following specific beam patterns and then demodulation with the simultaneously acquired excitation Cherenkov beam shapes is possible. Like the imaging technique used in CT (bottom), CELSI images were acquired by rotating the Linac gantry to yield a sinogram, which was then used with FBP of the data for image reconstruction. In all experiments, a plane mirror was always used to avoid direct exposure of the imaging camera to the radiation beam.

The recontruction can be improved by combining useful prior information about the measurment geometry, charateristic emissions, etc., into the regularization process, which has been an effective way, albeit with the assumption of the priors being accurate. ${ }^{84-90}$

By taking advantage of a careful measurement geometry and utilization of coded illumination techniques, a straightforward direct deconvolution has been used for reconstruction, e.g., Cherenkov-excited luminescence scanned imaging (CELSI). CELSI uses the collimating system [Fig. 4(a)] of a Linac to send a sheet of radiation traveling across the imaged subject in a manner equivalent to the excitation-beam shaping used in light-sheet microscopy, as illustrated in the first row of Fig. 5. By restricting the excitation beam to a single, narrow sheet, the origin of the optical photons can be inferred regardless of where these photons were detected or how many times they scattered in tissue. Direct deconvolution was also applied for a vertical illumination geometry as shown in the second row of Fig. 5. By simultaneously capturing both Cherenkov and CELSI images that were excited via spatially modulated $\mathrm{x}$-ray beam, the image recovery was improved by a spatial demodulation strategy at each time step based upon compressed sensing techniques. However, direct deconvolution typically suffers from two issues: (i) accurate convolutional kernels are generally hard to be determined with acceptable computational cost and (ii) numerical deconvolution could magnify the measurement noise when the amplitude approaches zero in the frequency domain.

Backprojection or filtered backprojection (FBP) reconstruction algorithm originally developed for CT has been used, known as X-ray luminescence CT (XLCT) ${ }^{3,91}$ Like the measurement geometry in CT, raw images in XLCT were acquired by rotating the Linac gantry (see the last row of Fig. 5) to render a sinogram that was used in backprojection-based image reconstruction. Each element in the sinogram is an integration through the pixels of a captured image, essentially implementing single-pixel imaging that facilitates the use of single-photon point detector for high-sensitivity measurement in conjunction with the TCSPC technique and the use of a spectrometer for hyperspectral studies. In XLCT, anatomical prior information can be provided by either X-ray imaging or Cherenkov surface data. ${ }^{44,92-94}$ Since the measurements are taken from multiple views, XLCT in-vivo imaging is similar to CT imaging in that the image is always better 
with more radiation, which can lead to excessive radiation dose, and so care must be taken to design the scan sequence. To address this issue, a lot of effort has focused on sparse-view XLCT by taking advantage of improved measurement geometry and algorithms. ${ }^{92,95,96}$

\section{Radioluminescent Reporters: Scintillators and Fluorophores}

The range of $\mathrm{x}$-ray choices, as described already, also affects the radioluminescent reporter agent that is optimally used, as the sources in the $\mathrm{keV}$ range excite scintillators exclusively, whereas the sources in the $\mathrm{MeV}$ range can excite all of fluorophores, phosphors, and scintillators.

Development of scintillating nanoparticles that can convert x-ray radiation into UV-vis-NIR light is a very active area of research. While there is incredible promise, all known scintillating reporters are in preclinical molecular imaging research or ex vivo use. ${ }^{97}$ With slight scattering and absorption of $\mathrm{x}$-ray in soft tissues, this type $\mathrm{x}$-ray-excited luminescence allows for deep in vivo optical imaging with ultrahigh spatial resolution and negligible tissue autofluorescence. ${ }^{4,98}$ Lanthanide-doped fluoride-based nanoparticles have a high atomic number and proper electronic energy states for downconversion of x-rays into UV-vis-NIR luminescence. ${ }^{98-101}$ $\mathrm{NaGdF} 4 \mathrm{Eu}^{3+} / \mathrm{Tb}^{3+}$ nanoparticles are a representative type, with efficient luminescence emission under $\mathrm{x}$-ray irradiation, because the emission energy transitions within $\mathrm{Gd}^{3+}$ can resonantly couple to the excited state of $\mathrm{Eu}^{3+} / \mathrm{Tb}^{3+}$ ions in the $\mathrm{Gd}^{3+}-\mathrm{Eu}^{3+} / \mathrm{Tb}^{3+}$ host-dopant combination. ${ }^{102-104}$ Lanthanide-doped oxide-based nanoparticles also show bright $\mathrm{x}$-ray-excited luminescence. ${ }^{105-108}$ For example, $\mathrm{Gd}_{2} \mathrm{O}_{2} \mathrm{~S}$ : Tb-based nanoparticles have been designed for $\mathrm{pH}$-dependent sensors, for monitoring bacterial infection or as nanocapsules that carry chemotherapy. ${ }^{109-111}$ Some scintillating nanomaterials in this category exhibit x-ray-excited persistent luminescence, which can still emit long-lasting phosphorescence after the x-ray irradiation is completed. ${ }^{106,112,113}$ Other composite nanomaterials, such as quantum dots (QDs), ${ }^{114}$ silicon nanocrystals, ${ }^{115}$ metal-organic structures, ${ }^{116}$ and gold nanoclusters, ${ }^{117}$ have also been reported to emit luminescence under $\mathrm{x}$-ray irradiation. A table containing a list of most molecules and nanoparticles used in vitro or in vivo is presented in Table 1 with a summary of each key discovery.

$\mathrm{MeV}$ x-ray-induced Cherenkov emission can be used as a controllable indirect light source that can scan the imaging objects to excite optical molecular probes, without the need for scintillation. ${ }^{41,80,81,120}$ While the overall yield of Cherenkov is low compared to scintillation, the attractive optical feature of Cherenkov is its broadband spectrum, ranging from UV through visible to NIR wavelengths,${ }^{51}$ which provides potential to excite almost all the optical molecular probes with absorption in this spectral region in theory. The challenge of this though is that the broadband signal introduces a spectral overlapping problem in the optical signal detection. To unmix the Cherenkov emission and the secondary optical emission signal, phosphorescent probes with long lifetimes have been used for Cherenkov-excited luminescence imaging with time gated acquisition. ${ }^{126}$ Alternatively, spectrally resolved detection based on spectrometer detection can capture the Cherenkov-excited fluorescence. ${ }^{58}$ In addition to this, SWIR (1000 to $1700 \mathrm{~nm}$ ) fluorophore PdSe QDs can also be used with Cherenkov-excited fluorescence imaging, to try to minimize the emission overlap based upon the longer Stokes-shift of the SWIR emitter. ${ }^{52}$ Perhaps the single most important feature of Cherenkov absorption is the fact that organic dyes can be used and therefore have a reasonably good potential for human use. In particular, emission is likely available from all FDA-approved fluorophores, such as fluorescein, methylene blue, and indocyanine green, although the emission quantum yield of the probe plays a big role in the efficiency of detection.

\section{Applications and Future Directions}

\subsection{Molecular Sensing of Oxygen and $\mathrm{pH}$}

Tumor oxygenation significantly affects the outcome of radiotherapy, and hypoxic tissues such as tumors (defined as having partial pressure of oxygen, $\mathrm{pO}_{2}$, less than $10 \mathrm{mmHg}$ ) are known to be more resistant to radiation damage than fully oxygenated tissue. ${ }^{127-129}$ Therefore, monitoring 
Pogue et al.: Review of in vivo optical molecular imaging and sensing from x-ray excitation

Table 1 In vitro or in vivo studies using or developing optical molecular probes for x-ray excitation.

\begin{tabular}{|c|c|c|c|c|c|}
\hline & Probes & Source & Appl. & Main results & Refs. \\
\hline \multirow[t]{4}{*}{1} & Oxyphor, G4, 2P & $\mathrm{MeV}$ & In vivo & $\begin{array}{l}\text { Tomographic imaging of } \mathrm{pO}_{2} \text { in deep } \\
\text { tissue using Cherenkov excitation with } \\
\text { radiotherapy. }\end{array}$ & 118,119 \\
\hline & & & & $\begin{array}{l}\text { Partial pressure of oxygen }\left(\mathrm{pO}_{2}\right) \text { in a } \\
\text { rat lymph node was imaged by } \\
\text { Cherenkov-excited luminescence } \\
\text { scans. }\end{array}$ & 120 \\
\hline & & & & $\begin{array}{l}\text { In vivo oxygenation imaging, defining } \\
\text { the resolution, depth, and sensitivity } \\
\text { limits for Cherenkov excitation scans. }\end{array}$ & 80 \\
\hline & & & & $\begin{array}{l}\text { In vivo mapping of tumor } \mathrm{pO}_{2} \\
\text { distributions with sub-mm spatial } \\
\text { resolution and tracking response to } \\
\text { radiotherapy. }\end{array}$ & 34 \\
\hline \multirow[t]{2}{*}{2} & Eu chelate microspheres & $\mathrm{MeV}$ & In vitro & $\begin{array}{l}\text { High-resolution Cherenkov-excited } \\
\text { luminescence scanning imaging } \\
\text { during a standard dynamic } \\
\text { radiotherapy. }\end{array}$ & 43 \\
\hline & & & In vivo & $\begin{array}{l}\text { Tomographic Cherenkov-excited } \\
\text { luminescence via multi-pinhole scan } \\
\text { approach for high-resolution in vivo } \\
\text { imaging. }\end{array}$ & 43 \\
\hline 3 & $\begin{array}{l}\text { IRDyes 680RD, } \\
\text { 700DX and } 800 \mathrm{CW}\end{array}$ & $\mathrm{MeV}$ & In vivo & $\begin{array}{l}\text { Cherenkov-excited fluorescence of } \\
\text { NIR IRDyes was successfully } \\
\text { detected by spectrally resolving } \\
\text { approach. }\end{array}$ & 58 \\
\hline 4 & PdSe QD & $\mathrm{MeV}$ & In vivo & $\begin{array}{l}\text { Cherenkov-excited SWIR, 1000- to } \\
\text { 1700-nm fluorescence imaging using } \\
\text { long Stokes-shift PdSe QDs. }\end{array}$ & 52 \\
\hline 5 & $\begin{array}{l}\mathrm{LaF}_{3}: \mathrm{Ce}^{3+}, \mathrm{Tb}^{3+} \\
\text { and } \mathrm{LaF}_{3}: \mathrm{Tb}^{3+}\end{array}$ & $\mathrm{keV}$ & In vitro & $\begin{array}{l}\text { Luminescence dominated by } \\
\text { emission from } \mathrm{Tb}^{3+} \text { ions and } \\
\text { enhanced by organic surface coating. }\end{array}$ & 99 \\
\hline 6 & Aerogel and Sylgard 184 & $\mathrm{keV}$ & In vivo & $\begin{array}{l}\text { Luminescence of composite silica } \\
\text { aerogels and Sylgard } 184 \text { and } \\
\mathrm{La}_{2} \mathrm{O}_{2} \mathrm{~S} \text { : Eu phosphor in } \\
\text { subcutaneous detection. }\end{array}$ & 105 \\
\hline 7 & $\mathrm{Sr}_{2} \mathrm{MgSi}_{2} \mathrm{O}_{7}: \mathrm{Eu}^{2+}, \mathrm{Dy}^{3+}$ & $\mathrm{keV}$ & In vitro & $\begin{array}{l}\text { Persistent luminescence where } \\
\text { characteristics are highly associated } \\
\text { with the synthesis conditions. }\end{array}$ & 121 \\
\hline 8 & $\mathrm{HfO}_{2}$ : Eu nanoparticles & $\mathrm{keV}$ & In vivo & $\begin{array}{l}\text { Bioinert nanoparticles for biological } \\
\text { luminescence imaging with excitation } \\
\text { by x-rays and UV-visible radiation. }\end{array}$ & 122 \\
\hline 9 & $\mathrm{NaGdF}_{4}: \mathrm{Tb} @ \mathrm{NaYF}_{4}$ & $\mathrm{keV}$ & In vivo & $\begin{array}{l}\text { Immunoassay tags for } \\
\text { autofluorescence-free high-sensitivity } \\
\text { detection of alpha-fetoprotein } \\
\text { biomarkers. }\end{array}$ & 104 \\
\hline 10 & $\mathrm{ZnGa2O} 4 \mathrm{Cr}^{3+}$ & $\mathrm{keV}$ & In vivo & $\begin{array}{l}\text { Delayed emission up to } 6 \mathrm{~h} \text {, at } 700 \mathrm{~nm} \\
\text { for in vivo whole body and tumor } \\
\text { imaging. }\end{array}$ & 106,112 \\
\hline 11 & $\begin{array}{c}\mathrm{NaLnF}_{4}: \mathrm{Tb}_{\mathrm{N}} @ \mathrm{NaYF}_{4} \\
\text { with BHQ1-DNA }\end{array}$ & $\mathrm{keV}$ & In vitro & $\begin{array}{l}\text { Nanocrystal scintillator-based } \\
\text { aptasensor to selectively sense } \\
\text { lysozymes in serum samples through } \\
\text { FRET. }\end{array}$ & 101 \\
\hline 12 & $\begin{array}{l}\mathrm{Lu}_{2} \mathrm{SiO}_{5}: \mathrm{Ce} \text { with } \\
\text { AlNap and AlRhod }\end{array}$ & $\mathrm{keV}$ & In vitro & $\begin{array}{l}\text { Luminescence tuned from blue to } \\
\text { green and red using FRET and able to } \\
\text { be successfully imaged in vitro with rat } \\
\text { cortical neurons. }\end{array}$ & 107 \\
\hline
\end{tabular}


Pogue et al.: Review of in vivo optical molecular imaging and sensing from x-ray excitation

Table 1 (Continued).

\begin{tabular}{|c|c|c|c|c|c|}
\hline & Probes & Source & Appl. & Main results & Refs. \\
\hline 13 & $\beta-\mathrm{NaGdF}_{4}: \mathrm{X} \% \mathrm{Eu}^{3+}$ & $\mathrm{keV}$ & In vivo & $\begin{array}{l}\text { Nanoparticles modified for high } \\
\text { luminescence intensity and ultralow } \\
\text { cytotoxicity, for in vivo x-ray } \\
\text { luminescence CT. }\end{array}$ & 91 \\
\hline 14 & $\begin{array}{l}\mathrm{NaLuF}_{4}: \mathrm{Gd}, \mathrm{Eu} @ \mathrm{Gd} \\
\text { Lu@Gd, Lu, Tb }\end{array}$ & $\mathrm{keV}$ & In vitro & $\begin{array}{l}\text { Excited by x-ray radiation for deep } \\
\text { tissue PDT and optical imaging, low } \\
\text { dark toxicity and effective } \\
\text { photocytotoxicity. }\end{array}$ & 100 \\
\hline 15 & $\begin{array}{c}\mathrm{Gd}_{2} \mathrm{O}_{2} \mathrm{~S} \text { : Eu scint-based } \\
\text { pH sensor }\end{array}$ & $\mathrm{keV}$ & In vitro & $\begin{array}{l}\text { An x-ray-excited luminescence-based } \\
\mathrm{pH} \text { sensor was fabricated to monitor } \\
\text { bacterial infection and treatment of } \\
\text { implanted devices. }\end{array}$ & 110,111 \\
\hline 16 & Au25-BSA cluster & $\mathrm{keV}$ & In vitro & $\begin{array}{l}\text { The x-ray-excited optical } \\
\text { luminescence of biomolecule-directed } \\
\text { metal clusters demonstrated. }\end{array}$ & 117 \\
\hline 17 & Hf-MOF and Zr-MOF & $\mathrm{keV}$ & In vitro & $\begin{array}{l}\text { Two metal organic frameworks } \\
\text { (MOFs) to efficiently convert x-ray to } \\
\text { visible-light luminescence were } \\
\text { designed. }\end{array}$ & 116 \\
\hline 18 & PEG-SrAl ${ }_{2} \mathrm{O}_{4}: \mathrm{Eu}^{2+}$ & $\mathrm{keV}$ & In vivo & $\begin{array}{l}\text { In vivo optical bioimaging in deep } \\
\text { tissues using soft } x \text {-ray-activated } \\
\text { persistent luminescence. }\end{array}$ & 113 \\
\hline 19 & $\mathrm{NaGdF}_{4}: \mathrm{Eu}^{3+}$ & $\mathrm{keV}$ & In vivo & $\begin{array}{l}\text { X-ray-excited luminescence and } \\
\text { photoluminescence, characterization } \\
\text { of crystal structure and extrinsic } \\
\text { factors. }\end{array}$ & 103 \\
\hline 20 & $\mathrm{Sr}_{8}\left(\mathrm{Si}_{4} \mathrm{O}_{12}\right) \mathrm{Cl}_{8}: \mathrm{Eu}^{2+}$ & $\mathrm{keV}$ & In vitro & $\begin{array}{l}\text { Temperature-dependent } \\
\text { radioluminescence, tested via UV light } \\
\text { and x-ray excitation. }\end{array}$ & 123 \\
\hline 21 & PEG-NaGd $\left(\mathrm{WO}_{4}\right)_{2}: \mathrm{Eu}$ & $\mathrm{keV}$ & In vivo & $\begin{array}{l}\text { Used as highly effective radio } \\
\text { luminescent nanoprobe for x-ray } \\
\text { optical imaging and contrast agent for } \\
\text { MRI and CT. }\end{array}$ & 124 \\
\hline 22 & Oxide-embedded Si-NCs & $\mathrm{keV}$ & In vitro & $\begin{array}{l}\text { The formation and x-ray luminescent } \\
\text { characterization of oxide-embedded } \\
\text { silicon nanocrystals (Si-NCs). }\end{array}$ & 115 \\
\hline 23 & DOX@Gd2O2S:T, @PSS/PAH & $\mathrm{keV}$ & In vivo & $\begin{array}{l}\text { Nanocapsules synthesized for } \\
\text { measuring } \mathrm{pH} \text {-triggered release of } \\
\text { doxorubicin with } \mathrm{x} \text {-rays. }\end{array}$ & 109 \\
\hline 24 & $\mathrm{BaGd}_{x} \mathrm{Y}_{1-} \mathrm{ZnO}_{5}: \mathrm{Yb}^{3+}$ & $\mathrm{keV}$ & In vivo & $\begin{array}{l}\text { Highly efficient x-ray-excited SWIR } \\
\text { luminescence phosphor for the deep- } \\
\text { tissue biological imaging. }\end{array}$ & 53 \\
\hline 25 & CdTe QDs & keV & In vivo & $\begin{array}{l}\text { Contrast in phantom and mouse tests, } \\
\text { quantified using clinical x-ray system } \\
\text { at } 20 \text { and } 120 \mathrm{keV} \text {. }\end{array}$ & 114 \\
\hline 26 & $\mathrm{Cs}_{2} \mathrm{NaY}_{0.99} \mathrm{~F}_{6}: 0.01 \mathrm{Pr}^{3+}$ & $\mathrm{keV}$ & In vitro & $\begin{array}{l}\text { Strong ultraviolet C ( } 200 \text { to } 280 \mathrm{~nm}) \\
\text { emission and afterglow for }>2-\mathrm{h} \text { post- } \\
\text { irradiation. }\end{array}$ & 125 \\
\hline 27 & $\mathrm{CeO}_{2}: \mathrm{Eu}^{3+}$ & $\mathrm{keV}$ & In vitro & $\begin{array}{l}\text { Red light emission excited with UV } \\
\text { light and x-ray. }\end{array}$ & 108 \\
\hline 28 & $\mathrm{Ba}_{0.55} \mathrm{Y}_{0.3} \mathrm{~F}_{2}: \mathrm{Eu}^{3+}$ & $\mathrm{keV}$ & In vivo & $\begin{array}{l}\text { Water-soluble cubic nanophosphors } \\
\text { surface modified for in vivo imaging } \\
\text { with } \beta+\text { from }{ }^{18} \mathrm{~F} \text { and } \mathrm{x} \text {-ray. }\end{array}$ & 98 \\
\hline
\end{tabular}




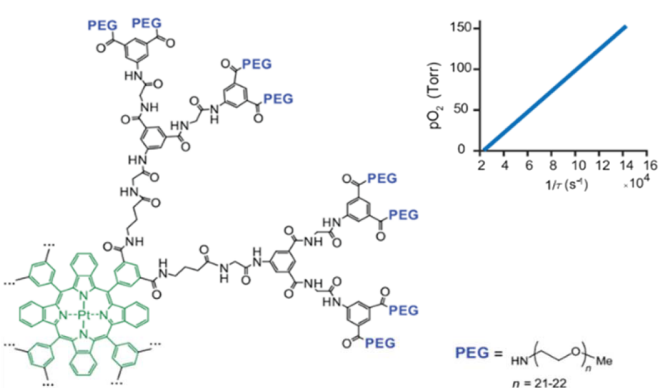

(a)
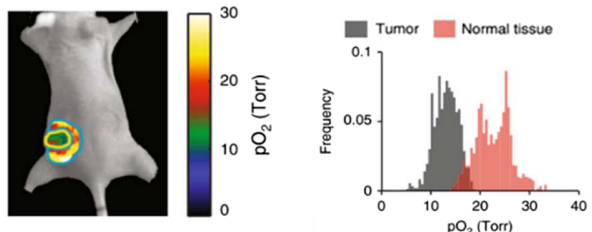

(b)

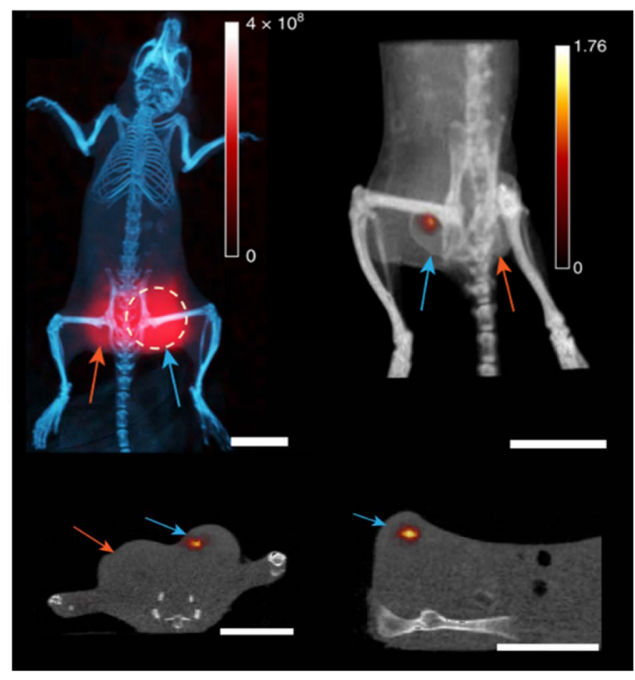

(c)

Fig. 6 Cherenkov-excited luminescence imaging of tumor oxygen. (a) Structure and oxygen quenching property of probe PtG4. ${ }^{34}$ (b) In vivo $\mathrm{pO}_{2}$ imaging of tumor and normal tissue of a mouse after IV injection of PtG4. ${ }^{34}$ (c) Maximum-intensity projection luminescence image overlaid to x-ray CT scan and tomographic luminescence of PtG4. ${ }^{80}$

of tumor oxygenation is thought to be highly desirable for effective radiotherapy. Cherenkovbased imaging provides an internal light source to excite oxygen-sensitive phosphorescence probes during radiotherapy (Fig. 6). ${ }^{34}$ Tomographic oxygen images were reconstructed in experimental mice, to sense the $\mathrm{pO}_{2} \cdot{ }^{118,119}$ Spatial resolution was improved by spatially encoding of the beam positioning with the Linac MLCs and subsequent deconvolution of the beam width from the signal. ${ }^{120,130}$ Using a scanning sheet-shaped MV x-ray beam, the excitation was restricted to narrow volumes, and tomographic oxygenation imaging with sub-millimeter resolution and nanomolar sensitivity at depths of several millimeters was demonstrated (Fig. 6). ${ }^{80}$ An alternative approach used the MV x-ray-induced Cherenkov emission spectral changes from differences in attenuation by the blood oxygen saturation, $\mathrm{SO}_{2}$ values. Tissue blood $\mathrm{SO}_{2}$ alters the absorption of the emitted Cherenkov, by de-oxygenated blood absorbing more in the 600- to $750-\mathrm{nm}$ wavelength band. The change in spectrum could be sampled, or more simply the change in broadband intensity could be tracked during radiation delivery. ${ }^{131,132}$

Another typical tumor microenvironment is the acidosis induced by high metabolic rate in poorly perfused regions of tumor, as a result extracellular $\mathrm{pH}$ in tumors is lower than in normal tissue and can be correlated with prognosis and response to treatment. ${ }^{133,134} \mathrm{An}$-ray-excited pH sensor consisted of an x-ray scintillator film $\left(\mathrm{Gd}_{2} \mathrm{O}_{2} \mathrm{~S}: \mathrm{Tb}\right.$ and $\left.\mathrm{Gd}_{2} \mathrm{O}_{2} \mathrm{~S}: \mathrm{Eu}\right)$ coated in a methyl red-dyed paper, used for high-resolution $\mathrm{pH}$ detection in tissue. The $\mathrm{pH}$ was obtained by analyzing the optical spectrum through the paper after scanning with narrow x-ray beam. ${ }^{135}$ Then the noninvasive $\mathrm{pH}$ sensing was used to monitor bacterial infection and treatment of implanted medical devices through tissues after coating on implanted surfaces. ${ }^{110,111}$ Using a pH-triggered nanocapsule, spectral changes were sensitive to the release of doxorubicin, which can be used to track drug release in the acidic tumor microenvironment. ${ }^{109}$ Some $\mathrm{pH}$-dependent long-lived emission luminescent probes appear promising for Cherenkov-based lifetime detection. ${ }^{136-138}$

\subsection{Sensing of Immunology: Biological Imaging and Development of Combined Therapeutics}

In the past few decades, the innovation of immunotherapy has revolutionized the approach to treatment of advanced cancer by means of immune cell therapy, stimulating the immune system to destroy malignant cells. ${ }^{139-141}$ Studies have used fluorescent imaging of NK cells within human prostate cancer xenografts ${ }^{142}$ and migration of T-cells to tumors. ${ }^{143,144}$ This fluorescent 
labeling technique for immune cells could be also used with x-rays to perform in vivo real-time monitoring of therapeutic effects for immune cell-based therapy. The relevance of this is because the combination of radiotherapy and immunotherapy is now shown to enhance the induced systemic anti-tumor response and achieve higher tumor control effect. ${ }^{145}$ Imaging has the potential to monitor immune signals during fractionated radiotherapy for patient specific sensing of the synergy between these treatments and their timing.

\subsection{Theranostics: Molecular Sensing with X-Ray-Induced Photodynamic Therapy}

The use of light as an activation mechanism has a long history in therapeutics, perhaps the most common being UV treatment for psoriasis ${ }^{146-149}$ and photodynamic therapy (PDT) ${ }^{150-152}$ PDT is a noninvasive cancer treatment modality that utilizes photosensitizers to generate cytotoxic reactive oxygen species when activated by light of appropriate wavelengths. ${ }^{153,154}$ PDT has its core strength in the shallow tissue penetration of illumination light, and this is widely utilized in dermatology for superficial skin lesion treatment. ${ }^{155-158}$ However, treatment of deep lesions has been limited by blood attenuation of the light. ${ }^{159-161}$ Using $\mathrm{X}$-rays as the irradiation source for PDT is a way to overcome this problem. This has been extensively studied in recent years, with many approaches using nanoscintillators to convert the x-rays to UV/visible light, which activates photosensitizer deep in tissues, ${ }^{162,163}$ and examples with direct Cherenkov activation are possible.

This approach to x-ray-activated photodynamic therapy (XPDT) makes it feasible to integrate diagnosis and tumor therapy for tumor theranostic applications. A theranostic mesoporous silica nanoparticle encapsulated a photosensitizer, 2,3-naphthalocyanine and a $\mathrm{LiGa}_{5} \mathrm{O}_{8}: \mathrm{Cr}$ based nanoscintillator was designed to efficiently mediate deep tissue XPDT and guide the irradiation by x-ray-excited luminescence imaging. ${ }^{164}$ This nanoparticle still produces ${ }^{1} \mathrm{O}_{2}$ from the long and intense afterglow luminescence of $\mathrm{LiGa}_{5} \mathrm{O}_{8}: \mathrm{Cr}$ after x-ray irradiation. After conjugation with Cetuximab (i.e., antibody to the EGF receptor), the nanoparticles were able to selectively accumulate in EGFR expressing orthotopic lung tumors for both EGFR-mediated molecular sensing with x-rays and XPDT. Another example constructed an x-ray-excited core-shell-shell theranostic scintillator nanoparticle based on lanthanides-doped $\mathrm{NaLuF}_{4}$, which could emit visible light, and was used with rose Bengal photosensitizer for PDT and imaging. ${ }^{100}$ Alternative approaches to drug release have used poly(lactide-co-glycolide) polymeric nanoparticles incorporating a photosensitizer (verteporfin) that could be triggered by $6-\mathrm{MeV} \mathrm{x}$-rays to generate ${ }^{1} \mathrm{O}_{2}$. In addition, targeting of nanoparticles with folic acid enables specific targeting of tumors that overexpress the folate receptors. Inclusion of radiation-activated $\mathrm{TiO}_{2}$ nanoparticles has been shown to have therapeutic effect, postulated to be mediated by Cherenkov light causing a photocatalytic effect, leading to radical species production. ${ }^{165}$

$\mathrm{X}$-ray deposited chemotherapies have been examined in several delivery moieties. Delivery via vitamin B12 uptake via the transcobalamin receptor was shown for delivery of agents and photorelease via the alkylcobalamin scaffold that is light sensitive. ${ }^{166} \mathrm{X}$-ray activation was shown to release doxorubicin bound in micelles, by breakage an o-nitrobenzyl linker, thereby breaking open micelles, and resulting in delivery of doxorubicin to the nucleus. ${ }^{167}$

While this last section has focused on therapeutic effects from x-rays mediated by optical signals, there is a linkage to diagnostic scanning that could be very important, both scientifically and practically. As these therapeutic studies advance, it seems inevitable that the diagnostic potential for their use will become more apparent as well.

\section{Conclusions}

Optical molecular sensing from x-ray excitation describes a range of technologies and research studies where an incident $\mathrm{x}$-ray beam is used for deep tissue sensing. The common theme is that through excitation by x-rays and active scanning or active delivery of molecular probes fundamentally new biological information could be sampled from tissue deeper than before and with higher spatial resolution. The field involves the intersection of (i) molecular probes that have 
high potential for radioluminescence or interaction with radioluminescence, (ii) x-ray technologies that provide specific energy, lateral or axial control, and scanning, and (iii) biomedical needs where there is not good potential for diagnostic information already. The strengths are in the widespread application and acceptance of x-rays as a diagnostic tool and the diversity of systems, energies, and controls that are well understood and developed. The challenges remain in the understanding and refinement of molecular probes that intake excitation energy from x-ray origins and maximize the output of optical signals in a way that retains meaningful molecular information by the location, intensity, or lifetime. While this field is not well defined as a single entity, it is inevitable that research will continue to define what is possible and that niche uses will become adopted, such as oxygen sensing, $\mathrm{pH}$ sensing, receptor uptake of nanomaterials, or X-PDT applications.

\section{Disclosures}

Brian Pogue and Petr Bruza report financial involvement with DoseOptics LLC, manufacturer of cameras and systems for Cherenkov imaging.

\section{Acknowledgments}

This work has been sponsored by the National Institutes of Health (Research Grant R01EB024498) and through the shared services of the Norris Cotton Cancer Center (Grant P30CA023108).

\section{Code, Data, and Materials Availability}

This work was a review of published material and so was not completed with any code, data, nor materials.

\section{References}

1. C. Carpenter et al., "Hybrid x-ray/optical luminescence imaging: characterization of experimental conditions," Med. Phys. 37(8), 4011-4018 (2010).

2. F. Leblond, K. M. Tichauer, and B. W. Pogue, "Singular value decomposition metrics show limitations of detector design in diffuse fluorescence tomography," Biomed. Opt. Express 1(5), 1514-1531 (2010).

3. G. Pratx et al., "Tomographic molecular imaging of x-ray-excitable nanoparticles," Opt. Lett. 35(20), 3345-3347 (2010).

4. G. Pratx et al., "X-ray luminescence computed tomography via selective excitation: a feasibility study," IEEE Trans. Med. Imaging 29(12), 1992-1999 (2010).

5. F. Leblond et al., "Pre-clinical whole-body fluorescence imaging: review of instruments, methods and applications," J. Photochem. Photobiol. B 98(1), 77-94 (2010).

6. K. K. Dwivedi et al., "Trace elemental analysis of extracted dust from lungs and lymph nodes of domestic animals using x-ray fluorescence technique," Int. J. Environ. Anal. Chem. 7(3), 205-221 (1980).

7. J. Borjesson and S. Mattsson, "Toxicology; in vivo x-ray fluorescence for the assessment of heavy metal concentrations in man," Appl. Radiat. Isot. 46(6-7), 571-576 (1995).

8. R. Zhang et al., "X-ray fluorescence imaging of metals and metalloids in biological systems," Am. J. Nucl. Med. Mol. Imaging 8(3), 169-188 (2018).

9. P. J. Riviere et al., "Accelerating x-ray fluorescence computed tomography," in Conf. Proc. IEEE Eng. Med. Biol. Soc., pp. 1000-1003 (2009).

10. S. K. Cheong et al., "X-ray fluorescence computed tomography (XFCT) imaging of gold nanoparticle-loaded objects using 110 kVp x-rays," Phys. Med. Biol. 55(3), 647-662 (2010).

11. M. Bazalova et al., "Investigation of x-ray fluorescence computed tomography (XFCT) and K-edge imaging," IEEE Trans. Med. Imaging 31(8), 1620-1627 (2012). 
Pogue et al.: Review of in vivo optical molecular imaging and sensing from x-ray excitation

12. Y. Kuang et al., "First demonstration of multiplexed x-ray fluorescence computed tomography (XFCT) imaging," IEEE Trans. Med. Imaging 32(2), 262-267 (2013).

13. D. Mustafi et al., "X-ray fluorescence microscopy demonstrates preferential accumulation of a vanadium-based magnetic resonance imaging contrast agent in murine colonic tumors," Mol Imaging 14 (5) (2015).

14. B. de Samber et al., "Three-dimensional elemental imaging by means of synchrotron radiation micro-XRF: developments and applications in environmental chemistry," Anal. Bioanal. Chem. 390(1), 267-271 (2008).

15. S. W. Price et al., "Chemical imaging of single catalyst particles with scanning mu-XANES-CT and mu-XRF-CT," Phys. Chem. Chem. Phys. 17(1), 521-529 (2015).

16. K. Langstraat et al., "Large area imaging of forensic evidence with MA-XRF," Sci. Rep. 7(1), 15056 (2017).

17. A. Turyanskaya et al., "Detection and imaging of gadolinium accumulation in human bone tissue by micro- and submicro-XRF," Sci. Rep. 10(1), 6301 (2020).

18. M. J. Pushie et al., "Elemental and chemically specific x-ray fluorescence imaging of biological systems," Chem. Rev. 114(17), 8499-8541 (2014).

19. L. Xiang et al., "X-ray acoustic computed tomography with pulsed x-ray beam from a medical linear accelerator," Med. Phys. 40(1), 010701 (2013).

20. L. Xiang et al., "High resolution X-ray-induced acoustic tomography," Sci. Rep. 6, 26118 (2016).

21. S. Hickling et al., "Experimental evaluation of x-ray acoustic computed tomography for radiotherapy dosimetry applications," Med. Phys. 44(2), 608-617 (2017).

22. P. Samant et al., "X-ray induced acoustic computed tomography," Photoacoustics 19, 100177 (2020).

23. Y. Zheng et al., "X-ray-induced acoustic computed tomography for guiding prone stereotactic partial breast irradiation: a simulation study," Med. Phys. 47, 4386-4395 (2020).

24. C. M. Carpenter et al., "Hybrid x-ray/optical luminescence imaging: characterization of experimental conditions," Med. Phys. 37(8), 4011-4018 (2010).

25. W. Cong et al., "X-ray micromodulated luminescence tomography in dual-cone geometry," J. Biomed. Opt. 19(7), 076002 (2014).

26. D. Chen et al., "Quantitative cone beam x-ray luminescence tomography/x-ray computed tomography imaging," Appl. Phys. Lett. 105(19), 191104 (2014).

27. X. Liu, Q. Liao, and H. Wang, "In vivo X-ray luminescence tomographic imaging with single-view data," Opt. Lett. 38(22), 4530-4533 (2013).

28. C. Li et al., "X-ray luminescence optical tomography imaging: experimental studies," Opt. Lett. 38(13), 2339-2341 (2013).

29. C. M. Carpenter et al., "Limited-angle x-ray luminescence tomography: methodology and feasibility study," Phys. Med. Biol. 56(12), 3487-3502 (2011).

30. M. C. Lun, W. Zhang, and C. Li, "Sensitivity study of x-ray luminescence computed tomography," Appl. Opt. 56(11), 3010-3019 (2017).

31. D. Chen et al., "Cone beam x-ray luminescence tomography imaging based on KA-FEM method for small animals," Biomed Res. Int. 2016, 6450124 (2016).

32. B. Meng, G. Pratx, and L. Xing, "Ultrafast and scalable cone-beam CT reconstruction using MapReduce in a cloud computing environment," Med. Phys. 38(12), 6603-6609 (2011).

33. R. Zhang et al., "Cherenkov-excited luminescence scanned imaging," Opt. Lett. 40(5), 827-30 (2015).

34. $\mathrm{X}$. Cao et al., "Tissue $\mathrm{pO}_{2}$ distributions in xenograft tumors dynamically imaged by Cherenkov-excited phosphorescence during fractionated radiation therapy," Nat. Commun. 11(1), 573 (2020).

35. H. A. Schmitt, D. G. Sample, and Y. M. Gu, "Pulsed radiography for detection of corrosion on shrink wrapped shipboard electrical connectors," Nav. Eng. J. 108(4), 35-40 (1996).

36. K. Santra et al., "What is the best method to fit time-resolved data? A comparison of the residual minimization and the maximum likelihood techniques as applied to experimental time-correlated, single-photon counting data," J. Phys. Chem. B 120(9), 2484-2490 (2016). 
37. P. Taroni et al., "Time-resolved diffuse optical spectroscopy: a differential absorption approach," Appl. Spectrosc. 64(11), 1220-1226 (2010).

38. W. Becker et al., "Fluorescence lifetime imaging by time-correlated single-photon counting," Microsc. Res. Tech. 63(1), 58-66 (2004).

39. X. Cao et al., "Time-gated luminescence imaging for background free in vivo tracking of single circulating tumor cells," Opt. Lett. 45(13), 3761-3764 (2020).

40. F. Romano et al., "Water-soluble silicon nanocrystals as NIR luminescent probes for timegated biomedical imaging," Nanoscale 12(14), 7921-7926 (2020).

41. M. J. Jia et al., "Multi-beam scan analysis with a clinical LINAC for high resolution Cherenkov-excited molecular luminescence imaging in tissue," Biomed. Opt. Express 9(9), 4217-4234 (2018).

42. E. P. M. LaRochelle and B. W. Pogue, "Theoretical lateral and axial sensitivity limits and choices of molecular reporters for Cherenkov-excited luminescence in tissue during x-ray beam scanning," J. Biomed. Opt. 26(11), 116004 (2020).

43. M. J. Jia et al., "Cherenkov-excited luminescence scanned imaging using scanned beam differencing and iterative deconvolution in dynamic plan radiation delivery in a human breast phantom geometry," Med. Phys. 46(7), 3067-3077 (2019).

44. M. J. Jia et al., "Tomographic Cherenkov-excited luminescence scanned imaging with multiple pinhole beams recovered via back-projection reconstruction," Opt. Lett. 44(7), 1552-1555 (2019).

45. S. Bajt et al., "X-ray focusing with efficient high-NA multilayer Laue lenses," Light Sci. Appl. 7, 17162 (2018).

46. F. Seiboth et al., "Perfect x-ray focusing via fitting corrective glasses to aberrated optics," Nat. Coтmun. 8, 14623 (2017).

47. H. Yumoto et al., "Ellipsoidal mirror for two-dimensional 100-nm focusing in hard x-ray region," Sci. Rep. 7(1), 16408 (2017).

48. Y. Bernhard, B. Collin, and R. A. Decreau, "Redshifted Cherenkov radiation for in vivo imaging: coupling Cherenkov radiation energy transfer to multiple Forster resonance energy transfers," Sci. Rep. 7, 45063 (2017).

49. R. S. Dothager et al., "Cerenkov radiation energy transfer (CRET) imaging: a novel method for optical imaging of PET isotopes in biological systems," PLoS One 5(10), e13300 (2010).

50. D. J. Naczynski et al., "X-ray-induced shortwave infrared biomedical imaging using rareearth nanoprobes," Nano Lett. 15(1), 96-102 (2015).

51. X. Cao et al., "Observation of short wavelength infrared (SWIR) Cherenkov emission," Opt. Lett. 43(16), 3854-3857 (2018).

52. X. Cao et al., "Cherenkov excited short-wavelength infrared fluorescence imaging in vivo with external beam radiation," J. Biomed. Opt. 24(5), 051405 (2018).

53. T. Li et al., "Efficient x-ray excited short-wavelength infrared phosphor," Opt. Express 27(9), 13240-13251 (2019).

54. X. Dai et al., "X-ray-induced shortwave infrared luminescence computed tomography," Opt. Lett. 44(19), 4769-4772 (2019).

55. H. Kantamneni et al., "Shortwave infrared emitting multicolored nanoprobes for biomarker-specific cancer imaging in vivo," BMC Cancer 20(1), 1082 (2020).

56. J. V. Shah et al., "Shortwave infrared-emitting theranostics for breast cancer therapy response monitoring," Front. Mol. Biosci. 7, 569415 (2020).

57. B. K. Byrd et al., "Characterizing short-wave infrared fluorescence of conventional nearinfrared fluorophores," J. Biomed. Opt. 24(3), 035004 (2019).

58. H. Lin et al., "Comparison of Cherenkov excited fluorescence and phosphorescence molecular sensing from tissue with external beam irradiation," Phys. Med. Biol. 61(10), 3955-3968 (2016).

59. A. Dsouza et al., "Cherenkov-excited multi-fluorophore sensing in tissue-simulating phantoms and in vivo from external beam radiotherapy," Radiat. Res. 189(2), 197-204 (2018).

60. A. R. Hounsell et al., "Computer-assisted generation of multi-leaf collimator settings for conformation therapy," Br. J. Radiol. 65(772), 321-326 (1992). 
Pogue et al.: Review of in vivo optical molecular imaging and sensing from x-ray excitation

61. E. J. Adams et al., "Comparison of a multi-leaf collimator with conformal blocks for the delivery of stereotactically guided conformal radiotherapy," Radiother. Oncol. 51(3), 205-209 (1999).

62. S. D. Randeniya et al., "Intercomparision of Monte Carlo radiation transport codes MCNPX, GEANT4, and FLUKA for simulating proton radiotherapy of the eye," Nucl. Technol. 168(3), 810-814 (2009).

63. H. Jiang and H. Paganetti, "Adaptation of GEANT4 to Monte Carlo dose calculations based on CT data," Med. Phys. 31(10), 2811-2818 (2004).

64. Q. Fang and D. A. Boas, "Monte Carlo simulation of photon migration in 3D turbid media accelerated by graphics processing units," Opt. Express 17(22), 20178-20190 (2009).

65. Q. Fang and S. Yan, "Graphics processing unit-accelerated mesh-based Monte Carlo photon transport simulations," J. Biomed. Opt. 24(11), 115002 (2019).

66. L. Jahnke et al., "GMC: a GPU implementation of a Monte Carlo dose calculation based on Geant4," Phys. Med. Biol. 57(5), 1217-1229 (2012).

67. T. Young-Schultz et al., "FullMonteCUDA: a fast, flexible, and accurate GPU-accelerated Monte Carlo simulator for light propagation in turbid media," Biomed. Opt. Express 10(9), 4711-4726 (2019).

68. T. S. Leung and S. Powell, "Fast Monte Carlo simulations of ultrasound-modulated light using a graphics processing unit," J. Biomed. Opt. 15(5), 055007 (2010).

69. J. Y. Cui et al., "Fully 3D list-mode time-of-flight PET image reconstruction on GPUs using CUDA," Med. Phys. 38(12), 6775-6786 (2011).

70. C. Thiam, C. Bobin, and J. Bouchard, "Simulation of Cherenkov photons emitted in photomultiplier windows induced by Compton diffusion using the Monte Carlo code GEANT4," Appl. Radiat. Isot. 68(7-8), 1515-1518 (2010).

71. D. Y. Shu et al., "Analysis on the emission and potential application of Cherenkov radiation in boron neutron capture therapy: a Monte Carlo simulation study," Appl. Radiat. Isot. 137, 219-224 (2018).

72. A. K. Glaser et al., "A GAMOS plug-in for GEANT4 based Monte Carlo simulation of radiation-induced light transport in biological media," Biomed. Opt. Express 4(5), 741-59 (2013).

73. E. P. M. LaRochelle, P. Arce, and B. W. Pogue, "Monte Carlo modeling photon-tissue interaction using on-demand cloud infrastructure," https://arxiv.org/abs/2005.01108 (2020).

74. E. Brost and Y. Watanabe, "Space-variant deconvolution of Cerenkov light images acquired from a curved surface," Med. Phys. 46(9), 4021-4036 (2019).

75. E. Brost and Y. Watanabe, "Characterization of the Cerenkov scatter function: a convolution kernel for Cerenkov light dosimetry," J. Biomed. Opt. 23(10), 105007 (2018).

76. A. K. Glaser et al., "Optical dosimetry of radiotherapy beams using Cherenkov radiation: the relationship between light emission and dose," Phys. Med. Biol. 59(14), 3789-3811 (2014).

77. A. K. Glaser et al., "Cherenkov radiation fluence estimates in tissue for molecular imaging and therapy applications," Phys. Med. Biol. 60(17), 6701-6718 (2015).

78. J. V. Jelley, Cerenkov Radiation and Its Applications, p. 304, Pergamon Press, New York (1958).

79. M. Cai et al., "NIR-II/NIR-I fluorescence molecular tomography of heterogeneous mice based on Gaussian weighted neighborhood fused Lasso method," IEEE Trans. Med. Imaging 39(6), 2213-2222 (2020).

80. B. W. Pogue et al., "Maps of in vivo oxygen pressure with submillimetre resolution and nanomolar sensitivity enabled by Cherenkov-excited luminescence scanned imaging," Nature Biomed. Eng. 2, 254-264 (2018).

81. P. Bruza et al., "Light sheet luminescence imaging with Cherenkov excitation in thick scattering media," Opt. Lett. 41(13), 2986-2989 (2016).

82. E. E. Brost and Y. Watanabe, "A mathematical deconvolution formulation for superficial dose distribution measurement by Cerenkov light dosimetry," Med. Phys. 45, 3880-3892 (2018). 
83. C. Li, A. Martinez-Davalos, and S. R. Cherry, "Numerical simulation of x-ray luminescence optical tomography for small-animal imaging," J. Biomed. Opt. 19(4), 046002 (2014).

84. J. Feng et al., "Multiobjective guided priors improve the accuracy of near-infrared spectral tomography for breast imaging," J. Biomed. Opt. 21(9), 090506 (2016).

85. G. Zhang et al., "A direct method with structural priors for imaging pharmacokinetic parameters in dynamic fluorescence molecular tomography," IEEE Trans. Biomed. Eng. 61(3), 986-990 (2014).

86. G. Zhang et al., "MAP estimation with structural priors for fluorescence molecular tomography," Phys. Med. Biol. 58(2), 351-372 (2012).

87. A. Ale et al., "Imaging performance of a hybrid x-ray computed tomography-fluorescence molecular tomography system using priors," Med. Phys. 37(5), 1976-1986 (2010).

88. C. Li et al., "A three-dimensional multispectral fluorescence optical tomography imaging system for small animals based on a conical mirror design," Opt. Express 17(9), 75717585 (2009).

89. A. Li et al., "Optimal linear inverse solution with multiple priors in diffuse optical tomography," Appl. Opt. 44(10), 1948-1956 (2005).

90. B. Brooksby et al., "Spectral priors improve near-infrared diffuse tomography more than spatial priors," Opt. Lett. 30(15), 1968-1970 (2005).

91. H. Zhang et al., "Combined multi-spectrum and orthogonal Laplacianfaces for fast CB-XLCT imaging with single-view data," Opt. Rev. 24, 693-704 (2017).

92. P. Gao et al., "Limited view cone-beam x-ray luminescence tomography based on depth compensation and group sparsity prior," J. Biomed. Opt. 26(1), 016004 (2020).

93. J. Shi et al., "Spatial weighed element based FEM incorporating a priori information on bioluminescence tomography," Lect. Notes Comput. Sci. 11(Pt. 1), 874-882 (2008).

94. H. Dehghani et al., "Spectrally resolved bioluminescence optical tomography," Opt. Lett. 31(3), 365-367 (2006).

95. T. Liu et al., "Regularized reconstruction based on joint L1 and total variation for sparseview cone-beam x-ray luminescence computed tomography," Biomed. Opt. Express 10(1), $1-17$ (2019).

96. X. Dai et al., "High-speed x-ray-induced luminescence computed tomography," J. Biophotonics 13, e202000066 (2020).

97. M. Nikl, "Wide band gap scintillation materials: progress in the technology and material understanding," Phys. Status Solidi A 178(2), 595-620 (2000).

98. C. Carpenter et al., "Limited-angle x-ray luminescence tomography: methodology and feasibility study," Phys. Med. Biol. 56(12), 3487 (2011).

99. Y. Liu et al., "X-ray luminescence of $\mathrm{LaF}_{3}: \mathrm{Tb}^{3+}$ and $\mathrm{LaF}_{3}: \mathrm{Ce}^{3+}, \mathrm{Tb}^{3+}$ water-soluble nanoparticles," J. Appl. Phys. 103(6), 063105 (2008).

100. C.-C. Hsu, S.-L. Lin, and C. A. Chang, "Lanthanide-doped core-shell-shell nanocomposite for dual photodynamic therapy luminescence imaging by a single $\mathrm{x}$-ray excitation source," ACS Appl. Mater. Interfaces 10(9), 7859-7870 (2018).

101. X. Ou et al., "X-ray nanocrystal scintillator-based aptasensor for autofluorescence-free detection," Anal. Chem. 91(15), 10149-10155 (2019).

102. W. Zhang et al., "Sub-10 nm water-dispersible $\beta-\mathrm{NaGdF}_{4} \mathrm{X} \% \mathrm{Eu}^{3+}$ nanoparticles with enhanced biocompatibility for in vivo x-ray luminescence computed tomography," ACS Appl. Mater. Interfaces 9(46), 39985-39993 (2017).

103. L. Sudheendra et al., " $\mathrm{NaGdF}_{4}: \mathrm{Eu}^{3+}$ nanoparticles for enhanced $\mathrm{x}$-ray excited optical imaging," Chem. Mater. 26(5), 1881-1888 (2014).

104. X.-Y. Ou et al., "Autofluorescence-free immunoassay using X-ray scintillating nanotags," Anal. Chem. 90(11), 6992-6997 (2018).

105. S. W. Allison et al., "In vivo x-ray excited optical luminescence from phosphordoped aerogel and Sylgard 184 composites," Radiat. Phys. Chem. 135, 88-93 (2017).

106. Z. Xue et al., "X-ray activated near-infrared persistent luminescent probe for deep-tissue and renewable in vivo bioimaging," ACS Appl. Mater. Interfaces 9(27), 22132-22142 (2017). 
107. M. K. Burdette et al., "Organic fluorophore coated polycrystalline ceramic LSO:Ce scintillators for x-ray bioimaging," Langmuir 35(1), 171-182 (2018).

108. S. Shi et al., "Solution combustion synthesis, photoluminescence and x-ray luminescence of Eu-doped nanoceria $\mathrm{CeO}_{2}$ :Eu," J. Mater. Chem. 22(44), 23461-23467 (2012).

109. H. Chen et al., "Monitoring $\mathrm{pH}$-triggered drug release from radioluminescent nanocapsules with x-ray excited optical luminescence," ACS Nano 7(2), 1178-1187 (2013).

110. F. Wang et al., "X-ray excited luminescence chemical imaging of bacterial growth on surfaces implanted in tissue," Adv. Healthcare Mater. 4(6), 903-910 (2015).

111. U. Uzair et al., "Noninvasively imaging PH at the surface of implanted orthopedic devices with x-ray excited luminescence chemical imaging," ACS Sens. 4(9), 2367-2374 (2019).

112. Z. Z. Chen et al., "Low dose of x-ray-excited long-lasting luminescent concave nanocubes in highly passive targeting deep-seated hepatic tumors," Adv. Mater. 31(49), 1905087 (2019).

113. L. Song et al., "Repeatable deep-tissue activation of persistent luminescent nanoparticles by soft x-ray for high sensitivity long-term in vivo bioimaging," Nanoscale 9(8), 27182722 (2017).

114. S. G. Ryan et al., "Imaging of x-ray-excited emissions from quantum dots and biological tissue in whole mouse," Sci. Rep. 9(1), 19223 (2019).

115. C. M. Hessel et al., "Origin of luminescence from silicon nanocrystals: a near edge x-ray absorption fine structure (NEXAFS) and x-ray excited optical luminescence (XEOL) study of oxide-embedded and free-standing systems," J. Phys. Chem. C 112(37), 14247-14254 (2008).

116. C. Wang et al., "Synergistic assembly of heavy metal clusters and luminescent organic bridging ligands in metal-organic frameworks for highly efficient x-ray scintillation," J. Am. Chem. Soc. 136(17), 6171-6174 (2014).

117. Y. Osakada et al., "Hard X-ray-induced optical luminescence via biomolecule-directed metal clusters," Chem. Commun. 50(27), 3549-3551 (2014).

118. R. Zhang et al., "Oxygen tomography by Cerenkov-excited phosphorescence during external beam irradiation," J. Biomed. Opt. 18(5), 050503 (2013).

119. R. W. Holt et al., "Cherenkov excited phosphorescence-based $\mathrm{pO}_{2}$ estimation during multi-beam radiation therapy: phantom and simulation studies," Phys. Med. Biol. 59(18), 5317-5328 (2014).

120. R. Zhang et al., "Cherenkov-excited luminescence scanned imaging," Opt. Lett. 40(5), $827-830$ (2015).

121. H. Homayoni et al., "X-ray excited luminescence and persistent luminescence of $\mathrm{Sr}_{2} \mathrm{MgSi}_{2} \mathrm{O}_{7}: \mathrm{Eu}^{2+}, \mathrm{Dy}^{3+}$ and their associations with synthesis conditions," J. Lumin. 198, 132-137 (2018).

122. J. Kaszewski et al., " $\mathrm{HfO}_{2}$ :Eu nanoparticles excited by $\mathrm{x}$-rays and UV-visible radiation used in biological imaging," J. Rare Earths 37(11), 1176-1182 (2019).

123. C. Liu et al., "High light yield of $\mathrm{Sr}_{8}\left(\mathrm{Si}_{4} \mathrm{O}_{12}\right) \mathrm{Cl}_{8}$ : $\mathrm{Eu}^{2+}$ under x-ray excitation and its temperature-dependent luminescence characteristics," Chem. Mater. 26(12), 3709-3715 (2014).

124. T. Guo et al., "High-efficiency $\mathrm{x}$-ray luminescence in $\mathrm{Eu}^{3+}$-activated tungstate nanoprobes for optical imaging through energy transfer sensitization," Nanoscale 10(4), 1607-1612 (2018).

125. Y.-M. Yang et al., "X-ray-activated long persistent phosphors featuring strong UVC afterglow emissions," Light Sci. Appl. 7(1), 1-11 (2018).

126. A. K. Glaser et al., "Time-gated Cherenkov emission spectroscopy from linear accelerator irradiation of tissue phantoms," Opt. Lett. 37(7), 1193-1195 (2012).

127. M. Hockel et al., "Hypoxia and radiation response in human tumors," Semin. Radiat. Oncol. 6(1), 3-9 (1996).

128. D. M. Brizel et al., "Oxygenation of head and neck cancer: changes during radiotherapy and impact on treatment outcome," Radiother. Oncol. 53(2), 113-117 (1999).

129. B. J Moeller, R. A. Richardson, and M. W. Dewhirst, "Hypoxia and radiotherapy: opportunities for improved outcomes in cancer treatment," Cancer Metastasis Rev. 26(2), 241-248 (2007). 
130. M. Jia et al., "Multi-beam scan analysis with a clinical LINAC for high resolution Cherenkov-excited molecular luminescence imaging in tissue," Biomed. Opt. Express 9(9), 4217-4234 (2018).

131. J. Axelsson et al., "Quantitative Cherenkov emission spectroscopy for tissue oxygenation assessment," Opt. Express 20(5), 5133-5142 (2012).

132. X. Zhang et al., "Noninvasive measurement of tissue blood oxygenation with Cerenkov imaging during therapeutic radiation delivery," Opt. Lett. 42(16), 3101-3104 (2017).

133. D. Neri and C. T. Supuran, "Interfering with $\mathrm{pH}$ regulation in tumours as a therapeutic strategy," Nat. Rev. Drug Discovery 10(10), 767-777 (2011).

134. F. A. Gallagher et al., "Magnetic resonance imaging of $\mathrm{pH}$ in vivo using hyperpolarized 13 C-labelled bicarbonate," Nature 453(7197), 940-943 (2008).

135. H. Chen et al., "High-resolution chemical imaging through tissue with an x-ray scintillator sensor," Anal. Chem. 83(13), 5045-5049 (2011).

136. S. Schreml et al., "2D luminescence imaging of $\mathrm{pH}$ in vivo," Proc. Natl. Acad. Sci. U.S.A. 108(6), 2432-2437 (2011).

137. M. Licini and J. G. Williams, "Iridium (III) bis-terpyridine complexes displaying longlived pH sensitive luminescence," Chem. Commun. (19), 1943-1944 (1999).

138. K. J. Arm, W. Leslie, and J. G. Williams, "Synthesis and pH-sensitive luminescence of bis-terpyridyl iridium (III) complexes incorporating pendent pyridyl groups," Inorg. Chim. Acta 359(4), 1222-1232 (2006).

139. P. Gangadaran and B.-C. Ahn, "Molecular imaging: a useful tool for the development of natural killer cell-based immunotherapies," Front. Immunol. 8, 1090 (2017).

140. J. Couzin-Frankel, "Cancer immunotherapy," Science 342(6165), 1432-1433 (2013).

141. S. J. Till et al., "Mechanisms of immunotherapy," J. Allergy Clin. Immunol. 113(6), 10251034 (2004).

142. S. Tavri et al., "Optical imaging of cellular immunotherapy against prostate cancer," Mol. Imaging 8(1), 7290.2009 (2009).

143. A. E. Foster et al., "In vivo fluorescent optical imaging of cytotoxic T lymphocyte migration using IRDye800CW near-infrared dye," Appl. Opt. 47(31), 5944-5952 (2008).

144. F. K. Swirski et al., "A near-infrared cell tracker reagent for multiscopic in vivo imaging and quantification of leukocyte immune responses," PLoS One 2(10), e1075 (2007).

145. M. B. Bernstein et al., "Immunotherapy and stereotactic ablative radiotherapy (ISABR): a curative approach?" Nat. Rev. Clin. Oncol. 13(8), 516-524 (2016).

146. P. C. Pasker-de Jong et al., "Treatment with UV-B for psoriasis and nonmelanoma skin cancer: a systematic review of the literature," Arch. Dermatol. 135(7), 834-840 (1999).

147. T. Mudigonda, T. S. Dabade, and S. R. Feldman, "A review of targeted ultraviolet B phototherapy for psoriasis," J. Am. Acad. Dermatol. 66(4), 664-672 (2012).

148. F. Almutawa et al., "Efficacy of localized phototherapy and photodynamic therapy for psoriasis: a systematic review and meta-analysis," Photodermatol. Photoimmunol. Photomed. 31(1), 5-14 (2015).

149. E. E. Bailey et al., "Combination treatments for psoriasis: a systematic review and metaanalysis," Arch. Dermatol. 148(4), 511-522 (2012).

150. Z. Huang, "A review of progress in clinical photodynamic therapy," Technol. Cancer Res. Treat. 4(3), 283-293 (2005).

151. B. C. Wilson and M. S. Patterson, "The physics, biophysics and technology of photodynamic therapy," Phys. Med. Biol. 53(9), R61-R109 (2008).

152. B. C. Wilson and M. S. Patterson, "The physics of photodynamic therapy," Phys. Med. Biol. 31(4), 327-360 (1986).

153. D. E. Dolmans, D. Fukumura, and R. K. Jain, "Photodynamic therapy for cancer," Nat. Rev. Cancer 3(5), 380-387 (2003).

154. P. Agostinis et al., "Photodynamic therapy of cancer: an update," CA Cancer J. Clin. 61(4), 250-281 (2011).

155. M. S. Nestor et al., "The use of photodynamic therapy in dermatology: results of a consensus conference," J. Drugs Dermatol. 5(2), 140-154 (2006).

156. S. R. Wiegell, "Update on photodynamic treatment for actinic keratosis," Curr. Probl. Dermatol. 46, 122-128 (2015). 
157. N. Solban et al., "Targeted optical imaging and photodynamic therapy," in Ernst Schering Res. Found Workshop, Vol. 49, pp. 229-58 (2005).

158. M. B. Ericson, A. M. Wennberg, and O. Larko, "Review of photodynamic therapy in actinic keratosis and basal cell carcinoma," Ther. Clin. Risk Manage. 4(1), 1-9 (2008).

159. Z. Fan et al., "eEF1A1 binds and enriches protoporphyrin IX in cancer cells in 5-aminolevulinic acid based photodynamic therapy," Sci. Rep. 6, 25353 (2016).

160. W. Chen and J. Zhang, "Using nanoparticles to enable simultaneous radiation and photodynamic therapies for cancer treatment," J. Nanosci. Nanotechnol. 6(4), 1159-1166 (2006).

161. H. Barr et al., "Review article: the potential role for photodynamic therapy in the management of upper gastrointestinal disease," Aliment. Pharmacol. Ther. 15(3), 311-321 (2001).

162. X. Zou et al., "X-ray-induced nanoparticle-based photodynamic therapy of cancer," Nanomedicine 9(15), 2339-2351 (2014).

163. H. Chen et al., "Nanoscintillator-mediated x-ray inducible photodynamic therapy for in vivo cancer treatment," Nano Lett. 15(4), 2249-2256 (2015).

164. W. Chen, W. Deng, and E. M. Goldys, "Light-triggerable liposomes for enhanced endolysosomal escape and gene silencing in PC12 cells," Mol. Ther. Nucleic Acids 7, 366-377 (2017).

165. N. Kotagiri et al., "Breaking the depth dependency of phototherapy with Cerenkov radiation and low-radiance-responsive nanophotosensitizers," Nat. Nanotechnol. 10(4), 370-379 (2015).

166. L. N. Gendron et al., "Tumor targeting vitamin B12 derivatives for x-ray induced treatment of pancreatic adenocarcinoma," Photodiagn. Photodyn. Ther. 30, 101637 (2020).

167. C. Yao et al., "X-ray-induced Cherenkov optical triggering of caged doxorubicin released to the nucleus for chemoradiation activation," ACS Appl. Mater. Interfaces 12(40), 4438344392 (2020).

Brian Pogue is the MacLean Professor of Engineering Science at Dartmouth, and an adjunct professor at the Geisel School of Medicine at Dartmouth. He works in optics in medicine and aspects of translational imaging work in surgery and radiation oncology.

Rongxiao Zhang is an assistant professor in medicine, within the Division of Radiation Oncology at Dartmouth, and has a research focus on novel tools and techniques for radiotherapy delivery, treatment planning, and verification.

Xu Cao is an assistant professor at Thayer School of Engineering, Dartmouth College. He obtained his $\mathrm{PhD}$ in biomedical engineering from Tsinghua University, China. His research focuses on optics in medicine, particularly Cherenkov excited luminescence imaging.

Jeremy Mengyu Jia is a postdoctoral researcher at Stanford University, having worked at Dartmouth College. His work is focused on optimization of image recovery and reconstruction imaging emerging imaging techniques.

Arthur Pétusseau is a PhD candidate in the Thayer School of Engineering at Dartmouth. His research interests are focused on time domain molecular imaging using fast-gated camera technologies. More specifically his work concentrates on x-ray excited organic fluorophores and their excitation mechanisms. In addition, he is working on in-vivo intracellular oxygen sensing using delayed fluorescence as well as time-of-flight depth sensing using an ultra-fast SPAD camera.

Petr Bruza is an assistant professor in engineering at Dartmouth, who specializes in radiation imaging and measurement, as well as photophysics and fast time-gated detection methods.

Sergei A. Vinogradov is a professor of biophysics and biochemistry at the University of Pennsylvania. His laboratory focuses on fundamentals of the chemistry and synthesis of molecular probes for sensing metabolically important features of tissue, such as oxygen. 